Article

\title{
Synthesis of A New Class of Pyridazin-3-one and 2-Amino-5- arylazopyridine Derivatives and Their Utility in the Synthesis of Fused Azines
}

\author{
Hamada Mohamed Ibrahim ${ }^{1,2}$ and Haider Behbehani ${ }^{1, *}$ \\ 1 Chemistry Department, Faculty of Science, Kuwait University, P.O. Box 5969, Safat 13060, \\ Kuwait; E-Mail: hamadaaldeb@yahoo.com \\ 2 Chemistry Department, Faculty of Science, Fayoum University, El-Fayoum 63514, A. R., Egypt \\ * Author to whom correspondence should be addressed; E-Mail: hidar@bahbahani.com; \\ Tel.: +965-990-630-62; Fax: +965-248-164-82.
}

Received: 27 January 2014; in revised form: 11 February 2014 / Accepted: 17 February 2014 / Published: 24 February 2014

\begin{abstract}
A general route for the synthesis of a novel class of pyridazin-3-one derivatives $\mathbf{3}$ by the reaction in acetic anhydride between 3-oxo-2-arylhydrazonopropanals 1 and some active methylene compounds like $p$-nitrophenylacetic acid and cyanoacetic acid was established. Under these conditions the pyridazin-3-one derivatives $\mathbf{3}$ were formed as the sole isolable products in excellent yield. The 6-acetyl-3-oxopyridazine derivative 31 was reacted with DMF-DMA to afford the corresponding enaminone derivative 4 , which reacts with a variety of aminoazoles to afford the corresponding azolo[1,5-a]pyrimidine derivatives 5-7. Also, in order to explore the viability and generality of a recently uncovered reaction between 3-oxo-2-arylhydrazonopropanals and active methylene compounds, a variety of 2-amino-6-aryl-5-arylazo-3-aroylpyridines 16-19 were prepared by reacting 3-oxo-2-arylhydrazonopropanals with miscellaneous active methylene compounds like 3-oxo-3-phenylpropionitrile, hetaroylacetonitriles and cyanoacetamides. These 2-aminopyridine derivatives undergo smooth reactions with cyanoacetic acid that led to the formation in high yield of a new class of 1,8-naphthyridine derivatives 24 . The structures of all new substances prepared in this investigation were determined by the different analytical spectroscopic methods, in addition to the X-ray crystallographic analysis.
\end{abstract}

Keywords: pyridazin-3-one; 2-amino-5-arylazopyridine; azolo[1,5-a]pyrimidine; DMF-DMA; cyanoacetic acid; 1,8-naphthyridine 


\section{Introduction}

Nitrogen-containing heterocyclic compounds have a diverse range of biological and pharmacological properties [1-3]. Pyridazine and pyridine derivatives are two of the most important heterocycles found in medicinal chemistry as they have an excellent biological activity with a wide range of applications, including antimicrobial [4-6], antiinflammatory and analgesic [7-9], anti-HIV [10], antiplasmodial [11], antitubercular [3,12], antibacterial [3,13], anticonvulsant [14,15], COX inhibitor [16], antidiabetic [17], antihypertensive [18,19], anticancer effects [20-24], blood platelet aggregation inhibitors [25], antidepressant and anxiolytic [26,27], antioxidant [28], antitumor [29,30] and antifungal activities [31]. For example Isoniazide and Amlodipine are two drugs containing the pyridine motif as anti-tuberculosis and anti-hypertensive respectively (Figure 1). On the basis of the above findings the pyridine and pyridazine moieties are considered privileged structures and, consequently, they have attracted the general and continuing interest of synthetic organic chemists.

Figure 1. Drugs containing the pyridine motif.<smiles>NNC(=O)c1ccncc1</smiles>

Isoniazide (anti-tuberculosis drug)<smiles>CCOCC1=C(C(=O)OCC)C(c2ccccc2Cl)C(C(C)=O)=C(C)N1</smiles>

Amlodipine (anti-hypertensive drug)

\section{Results and Discussion}

In earlier investigations we developed methods for the efficient synthesis of a variety of polyfunctional azoles, azines and their fused derivatives [32-36]. Recent efforts in our laboratories have led to the design of new and general strategies for the preparation of 2-amino-5-arylazonicotinates and pyridazinones [37] that involve reactions of 3-oxo-2-arylhydrazonopropanals 1 with active methylene compounds, including ethyl cyanoacetate, and malononitrile, depending on the effect of the substituent present in the arylazo moiety. Now it was of interest to explore the scope and limitations and generality of the 3-oxo-2-arylhydrazonopropanals $\mathbf{1}$ as a precursor for the synthesis of some new polyfunctionally substituted pyridazines and pyridines. In order to establish a general route for the synthesis of pyridazin-3-one derivatives $\mathbf{3}$ as sole products we conducted the reaction between 3-oxo-2-arylhydrazonopropanals 1 and some active methylene compounds, namely $p$-nitrophenylacetic acid (2a), o-nitrophenylacetic acid (2b) and cyanoacetic acid (2c) in acetic anhydride. Under these conditions only the pyridazin-3-one derivatives 3 were formed as sole isolable products in excellent yield. The structure of the pyridazin-3-one derivatives 3 was established based on their spectroscopic analyses and X-ray crystallographic analysis (Scheme 1, Figures 2 and 3). 
Scheme 1. Synthesis of pyridazin-3-one derivatives $\mathbf{3 a}-\mathbf{l}$.<smiles>[R]C(=O)/C(C=O)=N/N[3H]</smiles>

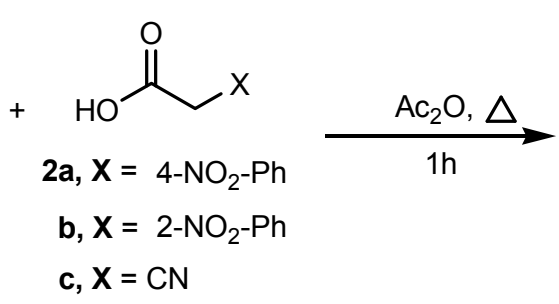<smiles>[R]C(=O)c1cc([X])c(=O)n([Al])n1</smiles>

\begin{tabular}{ccccc}
\hline Compound & $\mathbf{R}$ & $\mathbf{A r}$ & $\mathbf{X}$ & Yield \\
\hline $\mathbf{3 a}$ & $\mathrm{Ph}$ & $\mathrm{Ph}$ & $4-\mathrm{NO}_{2}-\mathrm{Ph}$ & $82 \%$ \\
$\mathbf{3 b}$ & $\mathrm{Ph}$ & $4-\mathrm{MeO}-\mathrm{Ph}$ & $4-\mathrm{NO}_{2}-\mathrm{Ph}$ & $84 \%$ \\
$\mathbf{3 c}$ & $\mathrm{Ph}$ & $4-\mathrm{Cl}-\mathrm{Ph}$ & $4-\mathrm{NO}_{2}-\mathrm{Ph}$ & $89 \%$ \\
$\mathbf{3 d}$ & $4-\mathrm{F}-\mathrm{Ph}$ & $\mathrm{Ph}$ & $4-\mathrm{NO}_{2}-\mathrm{Ph}$ & $80 \%$ \\
$\mathbf{3 e}$ & $4-\mathrm{F}-\mathrm{Ph}$ & $4-\mathrm{Cl}-\mathrm{Ph}$ & $4-\mathrm{NO}_{2}-\mathrm{Ph}$ & $84 \%$ \\
$\mathbf{3 f}$ & $4-\mathrm{Cl}-\mathrm{Ph}$ & $4-\mathrm{Cl}-\mathrm{Ph}$ & $4-\mathrm{NO}_{2}-\mathrm{Ph}$ & $78 \%$ \\
$\mathbf{3 g}$ & $4-\mathrm{Br}-\mathrm{Ph}$ & $4-\mathrm{Cl}-3-\mathrm{NO}_{2}-\mathrm{Ph}$ & $4-\mathrm{NO}_{2}-\mathrm{Ph}$ & $91 \%$ \\
$\mathbf{3 h}$ & $4-\mathrm{Br}-\mathrm{Ph}$ & $4-\mathrm{Cl}-3-\mathrm{NO}_{2}-\mathrm{Ph}$ & $4-\mathrm{NO}_{2}-\mathrm{Ph}$ & $77 \%$ \\
$\mathbf{3 i}$ & $4-\mathrm{Br}-\mathrm{Ph}$ & $4-\mathrm{Cl}-3-\mathrm{NO}_{2}-\mathrm{Ph}$ & $\mathrm{CN}^{2}$ & $75 \%$ \\
$\mathbf{3 j}$ & $4-\mathrm{Cl}-\mathrm{Ph}$ & $4-\mathrm{Cl}-\mathrm{Ph}$ & $\mathrm{CN}^{2}$ & $81 \%$ \\
$\mathbf{3 k}$ & $\mathrm{Ph}$ & $4-\mathrm{MeO}-\mathrm{Ph}$ & $\mathrm{CN}$ & $80 \%$ \\
$\mathbf{3 l}$ & $\mathrm{CH}$ & $\mathrm{Ph}$ & $4-\mathrm{NO}_{2}-\mathrm{Ph}$ & $89 \%$ \\
\hline
\end{tabular}

Figure 2. ORTEP plot of the X-ray crystallographic data determined for 3d [38].

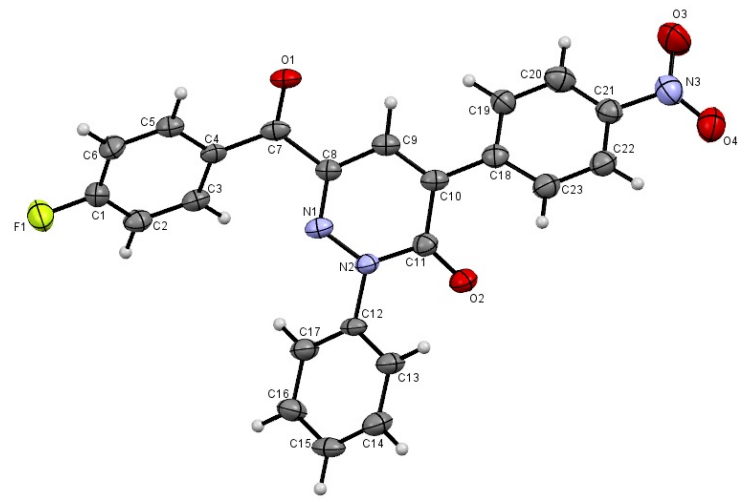

Figure 3. ORTEP plot of the X-ray crystallographic data determined for 31 [39].

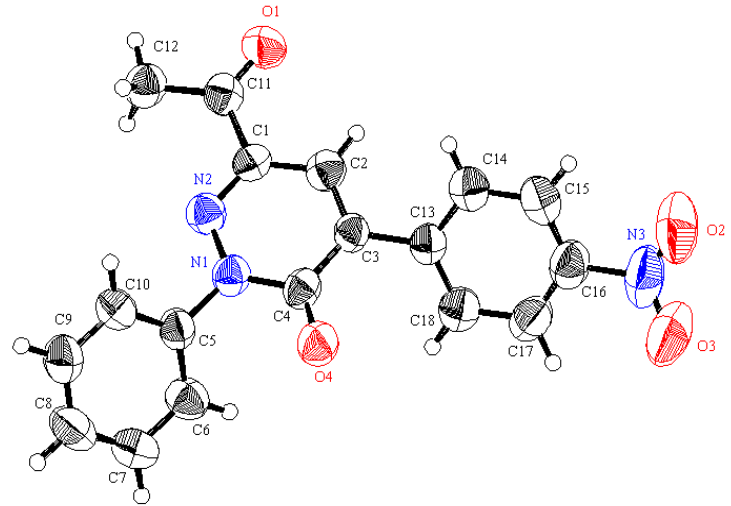


A plausible mechanism for the formation of pyridazin-3-ones 3 (Scheme 2) involves a condensation reaction between the two substrates $\mathbf{1}$ and $\mathbf{2}$ to generates the alkylidene intermediate $\mathbf{A}$, which then undergoes cyclization via elimination of another water molecule to afford smoothly the pyridazin-3ones 3. As illustrated in this mechanism, only two consecutive eliminations of water molecules in the presence of acetic anhydride as a reaction medium were needed to afford only the pyridazin-3-ones $\mathbf{3}$ in all cases and the formation of the 5-arylazopyridines not observed, due to the absence of ammonium acetate which furnishes the ammonia that plays an essential rule in the formation of the 5-arylazopyridines as described in previous studies $[37,40]$ and also in the forthcoming examples in this study.

Scheme 2. A plausible mechanism for the formation of pyridazin-3-ones 3 .

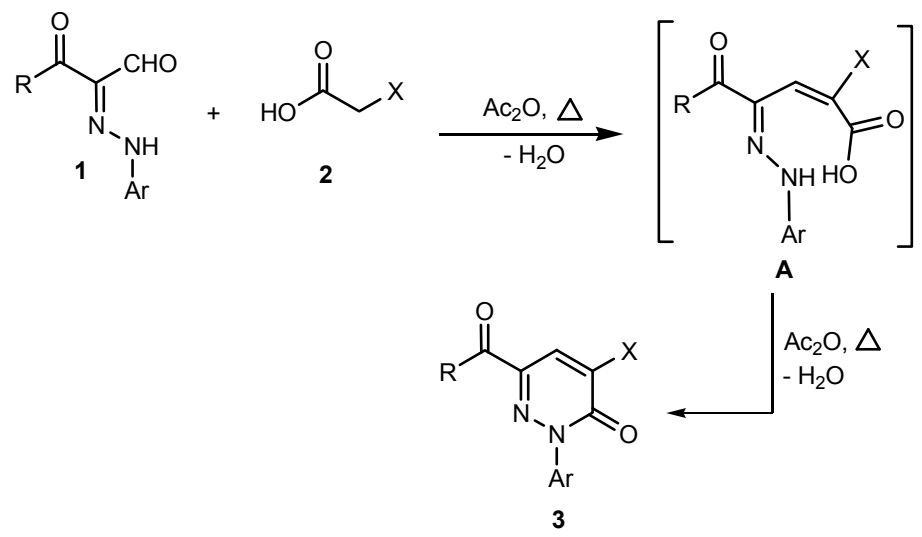

In order to synthesize a new class of enaminone derivatives the 6-acetyl-3-oxopyridazine derivative $\mathbf{3 l}$ was condensed with dimethylformamide dimethylacetal (DMF-DMA) in dioxane to yield the corresponding enaminone 4, whose ${ }^{1} \mathrm{H}-\mathrm{NMR}$ spectrum revealed the characteristic two doublet bands for the two olefinic CHs at $\delta 5.88$ and 7.81, respectively, and two signals due to the two methyl groups at $\delta$ 2.84 and 3.15, respectively. Moreover MS and HRMS showed its expected $\mathrm{M}^{+}$ion. The foregoing results prompted us to investigate the behaviour of the enaminone 4 towards some $N$-nucleophiles such as heterocyclic amines, as potential precursors of polyfunctionally-substituted fused pyrimidine derivatives for which we expect a broad spectrum of biological activity (Scheme 3).

Scheme 3. Reactions of the enaminone 4 with heterocyclic amines.

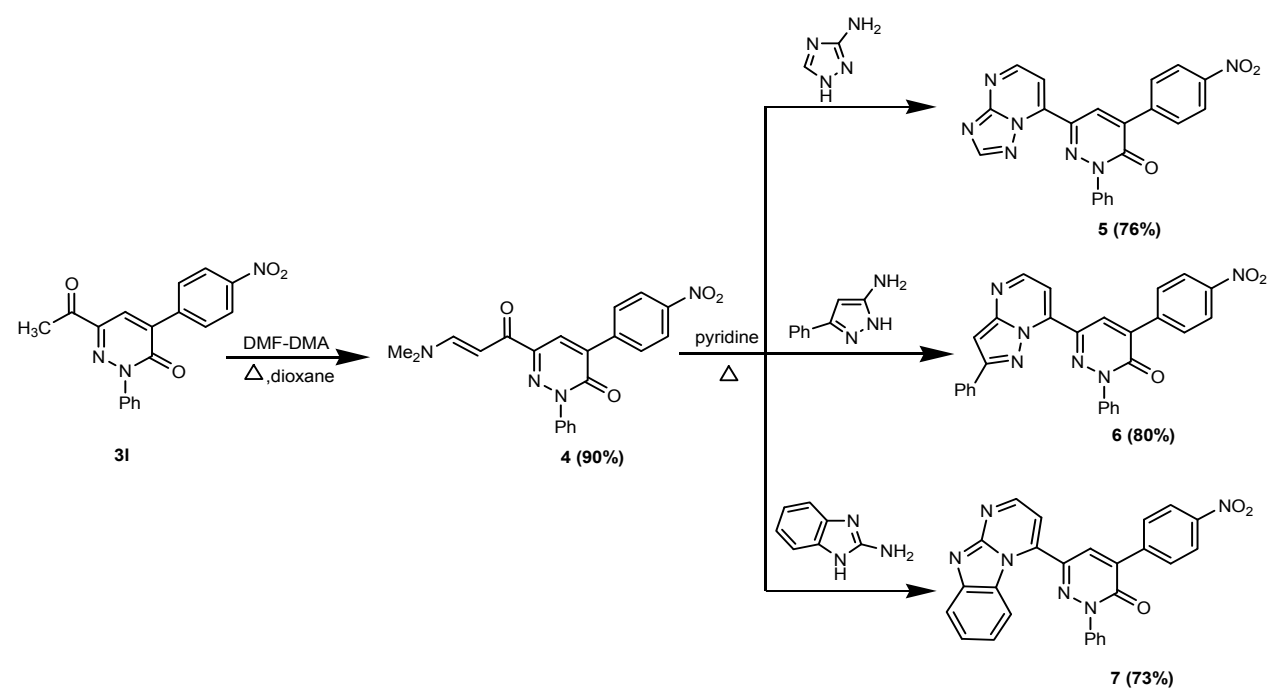


Thus the enaminone 4 was reacted with 3-amino-1,2,4-triazole, 3-phenyl-5-aminopyrazole and 2-aminobenzimidazole in refluxing pyridine to afford the corresponding azolo[1,5-a]pyrimidine derivatives 5-7 that incorporate the pyridazin-3-one moiety. A plausible mechanism for the formation of triazolo[1,5-a]pyrimidine derivatives $\mathbf{5}$ is taken as a representative example to explain the reaction between the enaminone 4 and heterocyclic amines (Scheme 4).

Scheme 4. A plausible mechanism for the formation of triazolo[1,5-a]pyrimidine derivatives 5 .

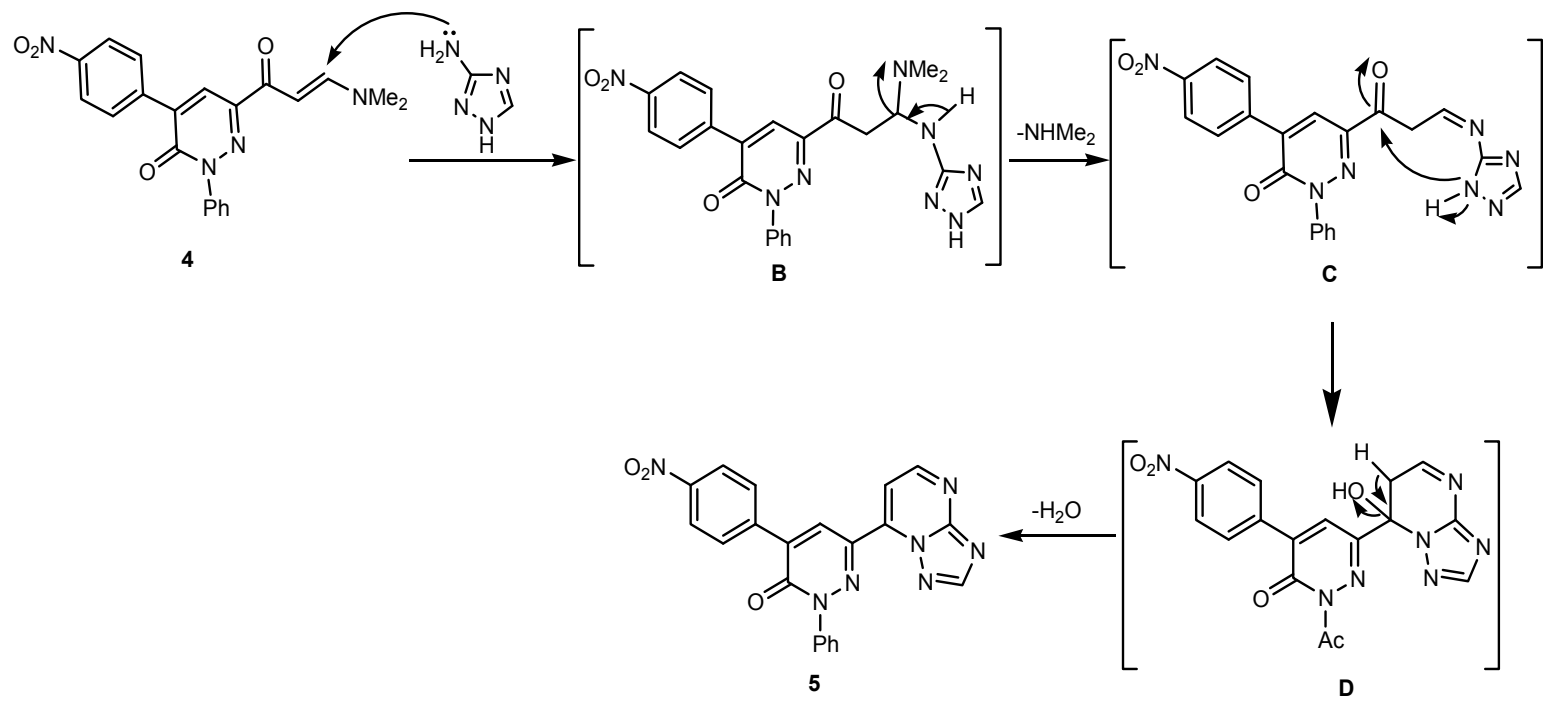

First a Michael-type addition of the exocyclic amino group in the aminotriazole to the $\alpha, \beta$-unsaturated moiety in the enaminone $\mathbf{4}$ yields the corresponding acyclic non-isolable intermediate $\mathbf{B}$, which forms the intermediate $\mathbf{C}$ via elimination of a dimethylamine molecule, and then the intermediate $\mathbf{C}$ undergoes cyclization through the addition of the $\mathrm{NH}$ to $\mathrm{CO}$ group to form the intermediate $\mathbf{D}$, followed by aromatization via loss of one water molecule to form finally the triazolo[1,5- $a]$ pyrimidine derivative $\mathbf{5}$.

In a recent study [40] we have also demonstrated that 2-amino-6-aryl-5-arylazo-3-benzoylpyridines 10 were formed as the sole isolable products in the reaction between 3-oxo-3-phenylpropionitrile (9) and 3-oxo-2-arylhydrazonopropanals 8a-b containing electron poor arylhydrazone groups as substrates, possessing two electron-withdrawing nitro and $\mathrm{Cl}$ groups on the aryl ring of this moiety (Scheme 5).

Scheme 5. Synthesis of 2-aminopyridines 10 [40].

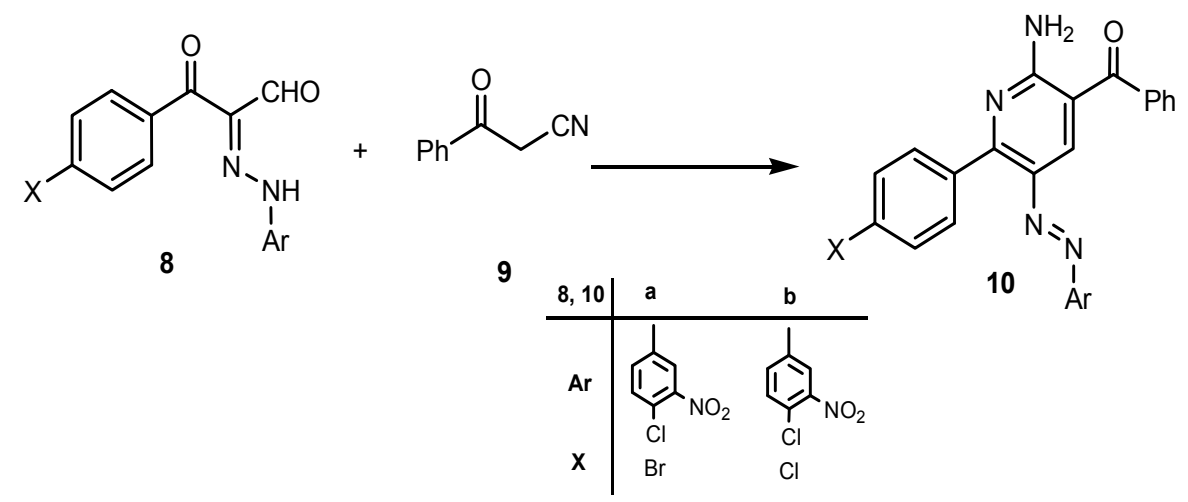


It was therefore of interest to explore the scope, limitations and extend the generality of reaction between 3-oxo-2-arylhydrazonopropanals 8a with miscellaneous active methylene compounds like 3-oxo-3-phenylpropionitrile, hetaroylacetonitriles and cyanoacetamides to afford polyfunctionally substituted 2-aminopyridines and their utility in the synthesis of 1,8-naphthyridine derivatives. Thus we explored reactions between 3-oxo-2-arylhydrazonopropanal 8a and cyanoacetylindoles 11,12 and different cyanoacetamides $\mathbf{1 3 , 1 4}$. These processes afford products which were shown to be the respective 2-aminopyridine derivatives 16-19 and it is believed that these 2-aminopyridines were formed via the intermediacy of $\mathbf{E}-\mathbf{G}$, as illustrated in the mechanistic pathway presented in (Scheme 6).

Scheme 6. A plausible mechanism for the formation of 2-aminopyridines.

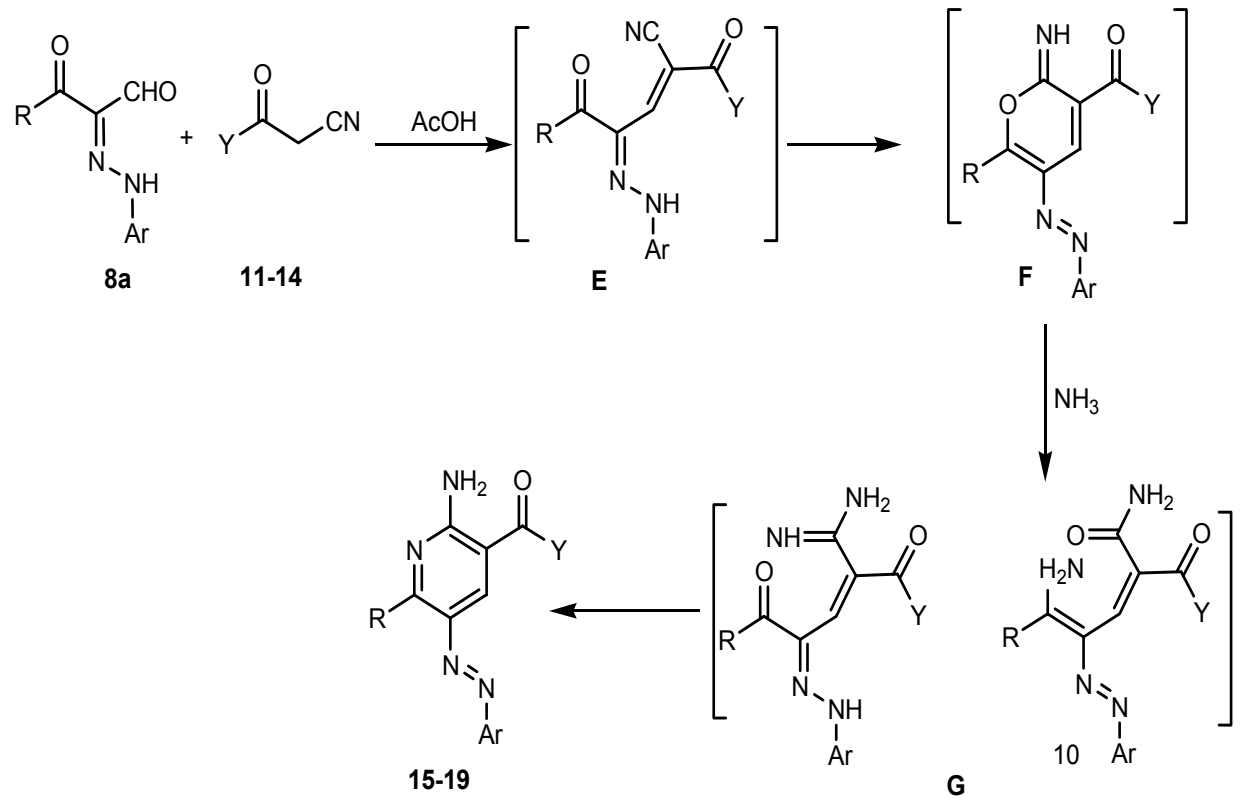

In contrast to the observed behaviour of 11-14 towards 8a the thiophene cyanoacetamide 15 behaves differently, affording the pyridazinimine derivatives $\mathbf{2 0}$ and not the 2-aminopyridine derivatives 21 or the 2-oxopyridines 22 according to the ${ }^{1} \mathrm{H}$-NMR spectra which showed two signals at $\delta \approx 9.80$ and $13.3 \mathrm{ppm}$ corresponding to two $\mathrm{NH}$ groups, one for the imine $\mathrm{NH}$ and the other for the amide $\mathrm{NH}$. Also the ${ }^{13} \mathrm{C}$-NMR spectrum showed a signal at $\delta \approx 187.5$ corresponding to a true ketone $\mathrm{CO}$ and not an amide $\mathrm{CO}$. Till now the factors that make the thiophene cyanoacetamide afford the pyridazine and not pyridine are not clear, but this behaviour may be related to the nature of the thiophene heterocyclic ring and is compatible with an earlier study in which when 3-oxo-2thiophenehydrazonopropanal reacts with ethyl cyanoacetate, it also affords the pyridazine and not the pyridine system [41]. The structures of these substances were assigned based on their spectroscopic and mass spectrometric properties and X-ray single crystal determination (Scheme 7, Figure 4).

In order to complete the goal of this study we conducted a reaction between the 2-aminopyridine derivatives 10a,b and cyanoacetic acid in the presence of acetic anhydride to smoothly afford the desired 1,8-naphthyridinecarbonitrile derivatives $\mathbf{2 4 a}, \mathbf{b}$ in very good yield. The reaction proceeds most likely via the intermediacy of $\mathbf{2 3}$ (Scheme 8). 
Scheme 7. Reaction of arylhydrazonopropanal 8a with miscellaneous active methylene compounds.

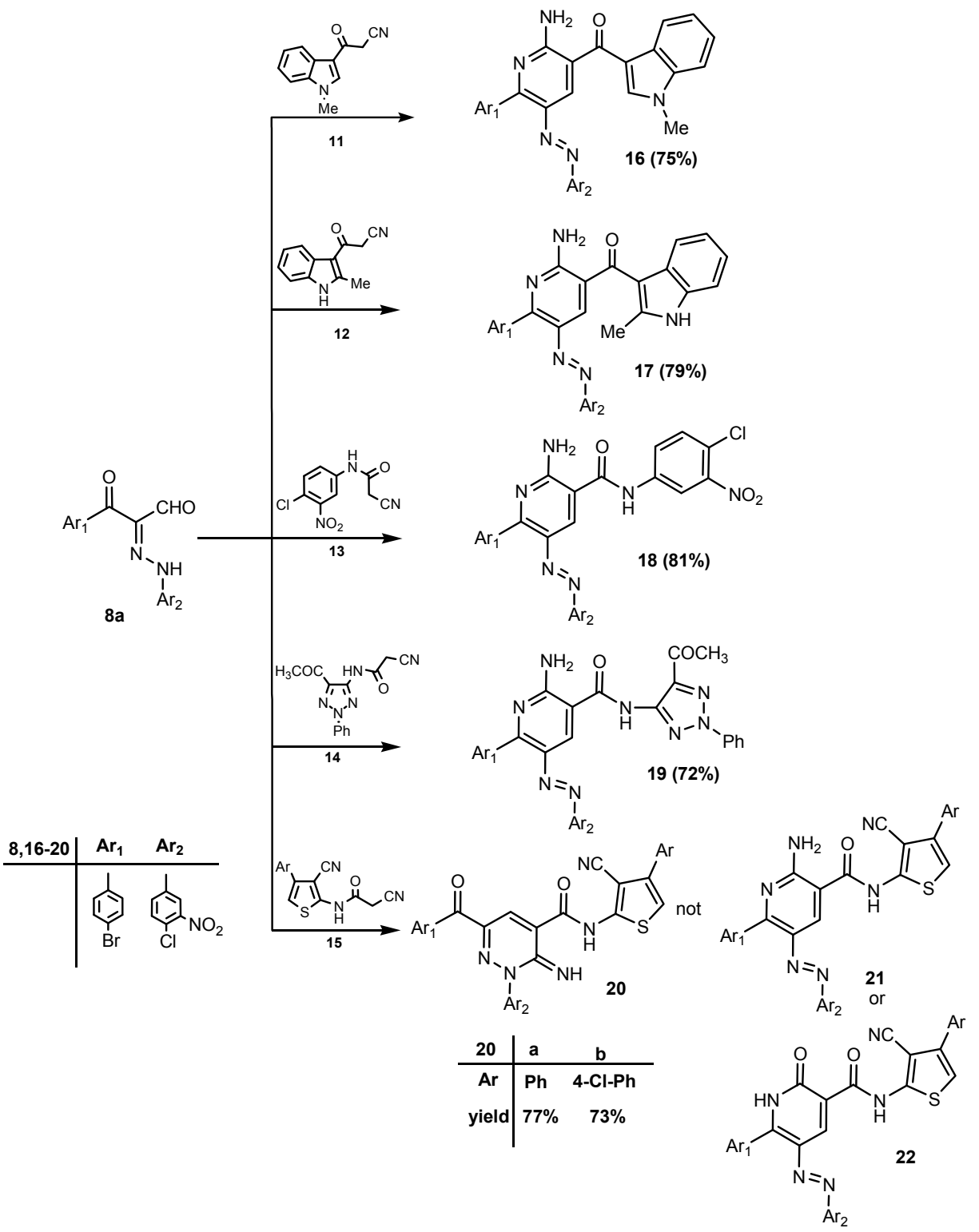

Figure 4. ORTEP plot of the X-ray crystallographic data determined for 18 [42].

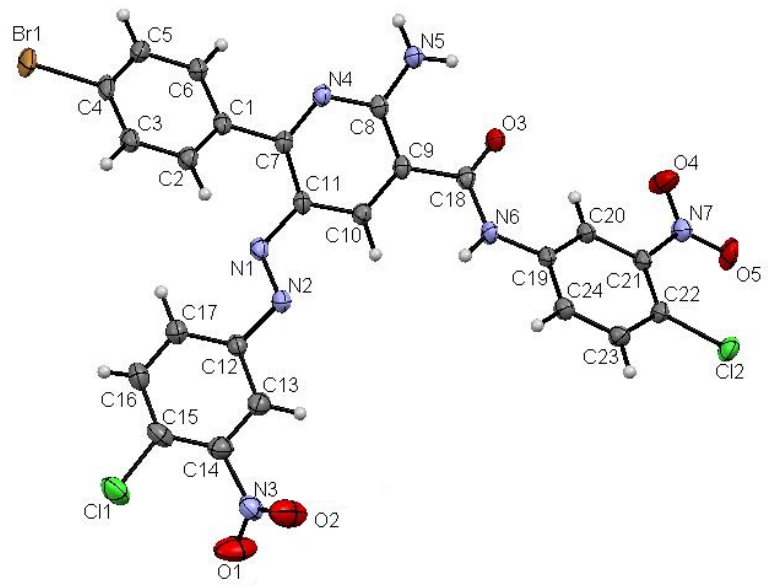


Scheme 8. Synthesis of 1,8-naphthyridines 24.

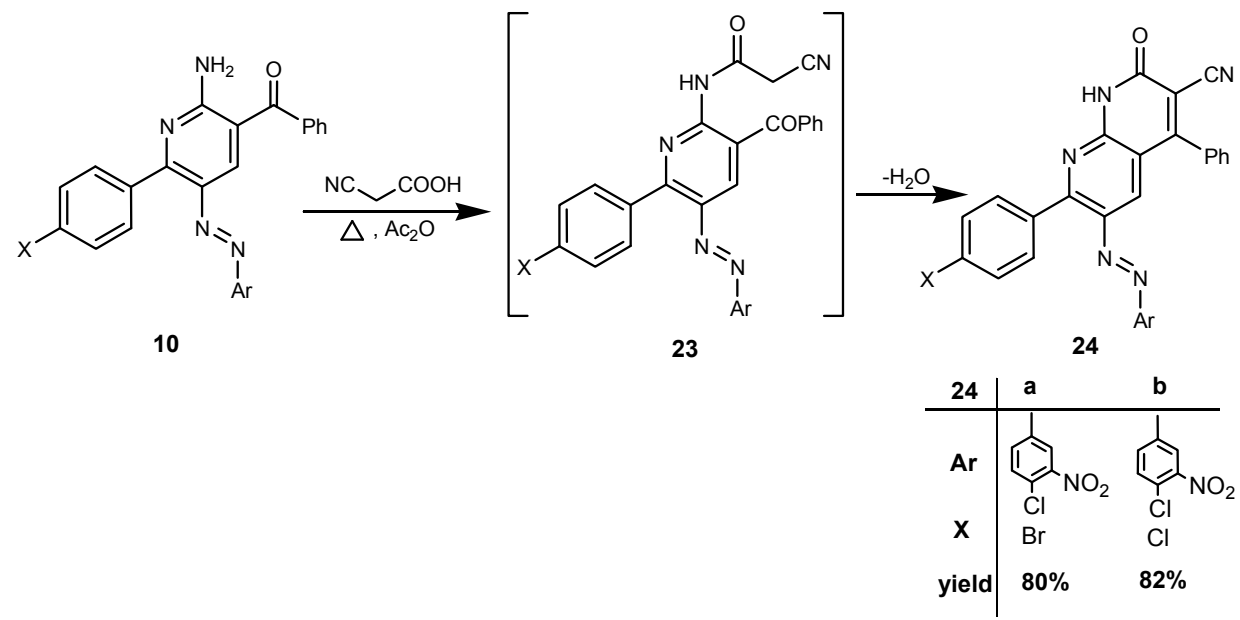

\section{Experimental}

\subsection{General}

Melting points were recorded and are reported uncorrected. The IR spectra were recorded using $\mathrm{KBr}$ pellets on a JASCO FTIR-6300 FT-IR spectrophotometer (Mary's Court, Easton, MD, USA). ${ }^{1} \mathrm{H}-\mathrm{NMR}(400 \mathrm{MHz})$ or $(600 \mathrm{MHz})$ and ${ }^{13} \mathrm{C}-\mathrm{NMR}(100 \mathrm{MHz})$ or $(150 \mathrm{MHz})$ spectra were recorded at $25{ }^{\circ} \mathrm{C}$ using $\mathrm{CDCl}_{3}$ or DMSO- $d_{6}$ solutions with TMS as an internal standard on a Bruker DPX 400 or 600 super-conducting NMR spectrometer (Rheinstetten, Germany). Chemical shifts are reported in ppm. Low-resolution electron impact mass spectra [MS (EI)] and high-resolution electron impact mass spectra [HRMS (EI)] were measured using a high resolution GC-MS (DFS) thermo spectrometer at $70.1 \mathrm{eV}$ using magnetic sector mass analyzer (Bremen, Germany). Microanalyses were performed on Elementar-Vario Micro cube Analyzer (Hanau, Germany). Monitoring reactions and determining the homogeneity of the prepared compounds were performed by using thin layer chromatography (TLC) (Sigma-Aldrich). The crystal structures were determined by a Rigaku R-AXIS RAPID diffractometer (Tokyo, Japan) and Bruker X8 Prospector (Madison, WI, USA) and the crystal data collections were made by using $\mathrm{Cu}-\mathrm{K} \alpha$ radiation. The data were collected at room temperature. The structure was solved by direct methods and was expanded using Fourier techniques. The non-hydrogen atoms were refined anisotropically. The structure was solved and refined using the Bruker SHELXTL Software Package (Structure solution program-SHELXS-97 and Refinement program-SHELXL-97) [43]. Data were corrected for the absorption effects using the multi-scan method (SADABS). Compounds 10a and $\mathbf{1 0 b}$ were prepared according to a literature procedure [40].

\subsection{Synthesis}

\subsubsection{General Procedure for the Preparation of Pyridazin-3-one Derivatives 3a-1}

Independent mixtures of $\mathbf{1 a}-\mathbf{l}(5 \mathrm{mmol}), p$-nitrophenylacetic acid $\mathbf{2 a}$ or cyanoacetic acid $\mathbf{2 b}(5 \mathrm{mmol})$, in acetic anhydride $(10 \mathrm{~mL})$ were stirred at reflux for $1 \mathrm{~h}$. The mixtures were cooled to room 
temperature. The formed solids were collected by filtration washed by EtOH and recrystallized from the appropriate solvent.

6-Benzoyl-4-(4-nitrophenyl)-2-phenylpyridazin-3-(2H)-one (3a). Yellow crystals, yield: (1.65 g, 82\%), m.p.: $182-183{ }^{\circ} \mathrm{C}$; IR (KBr): $v / \mathrm{cm}^{-1} 1672,1655$ (2CO); ${ }^{1} \mathrm{H}-\mathrm{NMR}$ (DMSO- $\left.d_{6}\right): \delta=7.50(\mathrm{t}, J=7.6 \mathrm{~Hz}$, 1H, Ar-H), 7.55-7.60 (m, 4H, Ar-H), 7.68-7.72 (m, 3H, Ar-H), 8.093 (d, J=8.0 Hz, 2H, Ar-H), 8.22 (d, $J=8.0 \mathrm{~Hz}, 2 \mathrm{H}, \mathrm{Ar}-\mathrm{H}), 8.29$ (s, $1 \mathrm{H}$, pyridazine $\mathrm{H} 5)$, and $8.37-8.39(\mathrm{~m}, 2 \mathrm{H}, \mathrm{Ar}-\mathrm{H}) ;{ }^{13} \mathrm{C}-\mathrm{NMR}$ $\left(\mathrm{DMSO}-d_{6}\right): \delta=123.9,126.6,128.9,129.3,129.4,129.43,130.8,131.1,133.9,135.8,137.9,140.5$, 142.1, 142.9, 148.4, 159.0 and $189.8 \mathrm{ppm}$ (Ar-C and CO); MS (EI): $\mathrm{m} / z$ (\%) $398\left([\mathrm{M}+1]^{+}, 28.15\right)$, $397\left(\mathrm{M}^{+}, 100\right)$; HRMS (EI): $m / z$ calcd. for $\mathrm{C}_{23} \mathrm{H}_{15} \mathrm{~N}_{3} \mathrm{O}_{4}\left(\mathrm{M}^{+}\right)$397.1057, found 397.1056. Anal. calcd. for $\mathrm{C}_{23} \mathrm{H}_{15} \mathrm{~N}_{3} \mathrm{O}_{4}$ (397.39): C, 69.52; H, 3.80; N, 10.57. Found: C, 69.43; H, 3.95; N, 10.66. (([M + 1 $]^{+}$ has be changed to $\left([\mathrm{M}+1]^{+}\right.$and please check)

6-Benzoyl-2-(4-methoxyphenyl)-4-(4-nitrophenyl)pyridazin-3-(2H)-one (3b). Yellow crystals, yield: (1.79 g, 84\%), m.p.: $167-168{ }^{\circ} \mathrm{C}$; IR (KBr): $v / \mathrm{cm}^{-1} 1670,1645$ (2CO); ${ }^{1} \mathrm{H}-\mathrm{NMR}$ (DMSO- $\left.d_{6}\right): \delta=3.83$ (s, 3H, $\mathrm{OCH}_{3}$ ), 7.09 (d, $\left.J=8.8 \mathrm{~Hz}, 2 \mathrm{H}, \mathrm{Ar}-\mathrm{H}\right), 7.58$ (t, $\left.J=8.0 \mathrm{~Hz}, 2 \mathrm{H}, \mathrm{Ar}-\mathrm{H}\right), 7.63$ (d, $J=8.8 \mathrm{~Hz}, 2 \mathrm{H}$, Ar-H), 7.70 (t, $J=8.0 \mathrm{~Hz}, 1 \mathrm{H}, \operatorname{Ar}-\mathrm{H}), 8.08$ (d, $J=8.0 \mathrm{~Hz}, 2 \mathrm{H}, \operatorname{Ar}-\mathrm{H}), 8.22$ (d, $J=8.8 \mathrm{~Hz}, 2 \mathrm{H}, \operatorname{Ar}-\mathrm{H})$, $8.27\left(\mathrm{~s}, 1 \mathrm{H}\right.$, pyridazine H5) and $8.38 \mathrm{ppm}(\mathrm{d}, J=8.8 \mathrm{~Hz}, 2 \mathrm{H}, \mathrm{Ar}-\mathrm{H}) ;{ }^{13} \mathrm{C}-\mathrm{NMR}\left(\mathrm{DMSO}-d_{6}\right): \delta=55.94$ $\left(\mathrm{CH}_{3}\right), 114.3,123.8,127.7,128.8,129.2,130.7,131.0,133.8,134.9,135.8,137.6,140.5,142.6,148.3$, 159.0, 159.6 and $189.8 \mathrm{ppm}$ (Ar-C and CO); MS (EI): $m / z(\%) 428\left([\mathrm{M}+1]^{+}, 34.22\right), 427\left(\mathrm{M}^{+}, 100\right)$; HRMS (EI): $m / z$ calcd. for $\mathrm{C}_{24} \mathrm{H}_{17} \mathrm{~N}_{3} \mathrm{O}_{5}\left(\mathrm{M}^{+}\right)$427.1162, found 427.1162 .

6-Benzoyl-2-(4-chlorophenyl)-4-(4-nitrophenyl)pyridazin-3-(2H)-one (3c). Pale orange crystals, yield: (1.9 g, 89\%), m.p.: $188-189{ }^{\circ} \mathrm{C}$; IR (KBr): $v / \mathrm{cm}^{-1} 1672,1639$ (2CO); ${ }^{1} \mathrm{H}-\mathrm{NMR}$ (DMSO- $\left.d_{6}\right): \delta=7.50$ (t, $J=8.0 \mathrm{~Hz}, 1 \mathrm{H}, \mathrm{Ar}-\mathrm{H}), 7.57$ (t, $J=8.0 \mathrm{~Hz}, 2 \mathrm{H}, \mathrm{Ar}-\mathrm{H}), 7.65$ (d, $J=8.8 \mathrm{~Hz}, 2 \mathrm{H}, \mathrm{Ar}-\mathrm{H}), 7.71$ (d, $J=8.0 \mathrm{~Hz}, 2 \mathrm{H}, \mathrm{Ar}-\mathrm{H}), 8.10(\mathrm{~d}, J=8.8 \mathrm{~Hz}, 2 \mathrm{H}, \mathrm{Ar}-\mathrm{H}), 8.21$ (d, $J=8.8 \mathrm{~Hz}, 2 \mathrm{H}, \mathrm{Ar}-\mathrm{H}), 8.27$ (s, 1H, pyridazine $\mathrm{H} 5$ ) and $8.38 \mathrm{ppm}(\mathrm{d}, J=8.8 \mathrm{~Hz}, 2 \mathrm{H}, \mathrm{Ar}-\mathrm{H}) ;{ }^{13} \mathrm{C}-\mathrm{NMR}$ (DMSO- $\left.d_{6}\right): \delta=123.9,126.5$, 129.0, 129.23, 129.24, 129.3, 130.7, 132.9, 134.4, 137.9, 138.8, 140.3, 141.9, 142.6, 148.3, 158.9 and $188.6 \mathrm{ppm}$ (Ar-C and CO); MS (EI): $m / z(\%) 432$ ([M + 1] $\left.]^{+}, 35.87\right), 431\left(\mathrm{M}^{+}, 100\right)$; HRMS (EI): $m / z$ calcd. for $\mathrm{C}_{23} \mathrm{H}_{14}{ }^{35} \mathrm{ClN}_{3} \mathrm{O}_{4}\left(\mathrm{M}^{+}\right) 431.0667$, found 431.0669 .

6-(4-Fluorobenzoyl)-4-(4-nitrophenyl)-2-phenylpyridazin-3-(2H)-one (3d). Yellow crystals, yield: (1.7 g, 80\%), m.p.: $177-178{ }^{\circ} \mathrm{C}$; IR (KBr): $v / \mathrm{cm}^{-1} 1677,1655$ (2CO); ${ }^{1} \mathrm{H}-\mathrm{NMR}$ (DMSO- $\left.d_{6}\right): \delta=7.39(\mathrm{t}$, $J=8.4 \mathrm{~Hz}, 2 \mathrm{H}, \mathrm{Ar}-\mathrm{H}), 7.47-7.54$ (m, 3H, Ar-H), 7.68 (d, $J=8.8 \mathrm{~Hz}, 2 \mathrm{H}, \mathrm{Ar}-\mathrm{H}), 8.15-8.18$ (m, 4H, Ar-H), $8.24(\mathrm{~s}, 1 \mathrm{H}$, pyridazine $\mathrm{H} 5)$ and $8.34 \mathrm{ppm}(\mathrm{d}, J=8.8 \mathrm{~Hz}, 2 \mathrm{H}, \mathrm{Ar}-\mathrm{H}) ;{ }^{13} \mathrm{C}-\mathrm{NMR}$ (DMSO- $\left.d_{6}\right)$ : $\delta=115.9,116.1,123.8,126.5,129.2,129.3,130.7,132.3,134.03,134.10,137.8,140.3,142.0,142.7$, 148.3, 158.9, 164.8, 166.4 and $188.2 \mathrm{ppm}$ (Ar-C and CO); MS (EI): $m / z(\%) 416\left([\mathrm{M}+1]^{+}, 29.69\right)$, $415\left(\mathrm{M}^{+}, 100\right)$; HRMS (EI): $\mathrm{m} / z$ calcd. for $\mathrm{C} 23 \mathrm{H} 14 \mathrm{FN} 3 \mathrm{O} 4\left(\mathrm{M}^{+}\right)$415.0962, found 415.0963. Crystal Data, $\mathrm{C}_{23} \mathrm{H}_{14} \mathrm{~F} \mathrm{~N}_{3} \mathrm{O}_{4}, \mathrm{M}=415.38$, triclinic, $\mathrm{a}=7.751(3) \AA, \mathrm{b}=11.283(4) \AA, \mathrm{c}=22.132(8) \AA$, $\mathrm{V}=1873(1) \AA^{3}, \alpha=90.895(7)^{\circ}, \beta=97.459(7)^{\circ}, \gamma=102.263(8)^{\circ}$, space group: $\mathrm{P}-1(\# 2), \mathrm{Z}=4$, $\mathrm{D}_{\text {calc }}=1.473 \mathrm{~g} \cdot \mathrm{cm}^{-3}$, No. of reflection measured 8398, $2 \theta_{\max }=54.8^{\circ}, \mathrm{R} 1=0.0738$ [38]. 
2-(4-Chlorophenyl)-6-(4-fluorobenzoyl)-4-(4-nitrophenyl)pyridazin-3-(2H)-one (3e). Yellow crystals, yield: $\left(1.9\right.$ g, 84\%), m.p.: $214-215{ }^{\circ} \mathrm{C}$; IR (KBr): $v / \mathrm{cm}^{-1} 1675,1643$ (2CO); ${ }^{1} \mathrm{H}-\mathrm{NMR}$ (DMSO- $d_{6}$ ): $\delta=7.37-7.40(\mathrm{~m}, 2 \mathrm{H}, \mathrm{Ar}-\mathrm{H}), 7.60$ (d, $J=8.4 \mathrm{~Hz}, 2 \mathrm{H}, \mathrm{Ar}-\mathrm{H}), 7.72(\mathrm{~d}, J=8.4 \mathrm{~Hz}, 2 \mathrm{H}, \mathrm{Ar}-\mathrm{H}), 8.16-8.18$ $(\mathrm{m}, 4 \mathrm{H}, \mathrm{Ar}-\mathrm{H}), 8.23(\mathrm{~s}, 1 \mathrm{H}$, pyridazine $\mathrm{H} 5)$ and $8.33 \mathrm{ppm}(\mathrm{d}, J=8.4 \mathrm{~Hz}, 2 \mathrm{H}, \mathrm{Ar}-\mathrm{H}) ;{ }^{13} \mathrm{C}-\mathrm{NMR}$ $\left(\mathrm{DMSO}-d_{6}\right): \delta=115.9,116.1,123.8,128.4,129.3,130.7,132.2,133.7,134.05,134.11,137.9,140.2$, 140.7, 142.9, 148.3, 158.8, 164.8, 166.5 and $188.1 \mathrm{ppm}$ (Ar-C and CO); MS (EI): $m / z(\%) 450$ ([M + 1] , 24.91), $449\left(\mathrm{M}^{+}, 66.15\right)$; HRMS (EI): $m / z$ calcd. for $\mathrm{C}_{23} \mathrm{H}_{13}{ }^{35} \mathrm{ClFN}_{3} \mathrm{O}_{4}\left(\mathrm{M}^{+}\right) 449.0573$, found 449.0573 .

6-(4-Chlorobenzoyl)-2-(4-chlorophenyl)-4-(4-nitrophenyl)pyridazin-3-(2H)-one (3f).Yellow crystals, yield: $\left(1.8 \mathrm{~g}, 78 \%\right.$ ), m.p.: $256-257{ }^{\circ} \mathrm{C}$; IR (KBr): $v / \mathrm{cm}^{-1} 1670,1648$ (2CO); ${ }^{1} \mathrm{H}-\mathrm{NMR}$ (DMSO- $d_{6}$ ): $\delta=7.60-7.62(\mathrm{~m}, 4 \mathrm{H}, \mathrm{Ar}-\mathrm{H}), 7.72(\mathrm{~d}, J=8.4 \mathrm{~Hz}, 2 \mathrm{H}, \mathrm{Ar}-\mathrm{H}), 8.07$ (d, $J=8.4 \mathrm{~Hz}, 2 \mathrm{H}, \mathrm{Ar}-\mathrm{H}), 8.17$ (d, $J=8.4 \mathrm{~Hz}, 2 \mathrm{H}, \mathrm{Ar}-\mathrm{H}), 8.24$ (s, $1 \mathrm{H}$, pyridazine $\mathrm{H} 5)$ and $8.34 \mathrm{ppm}(\mathrm{d}, J=8.4 \mathrm{~Hz}, 2 \mathrm{H}, \mathrm{Ar}-\mathrm{H}) ;{ }^{13} \mathrm{C}-\mathrm{NMR}$ (DMSO- $d_{6}$ ): $\delta=123.9,128.3,129.0,129.25,129.30,130.7,132.9,133.7,134.3,138.0,138.88,140.2$, 140.7, 142.7, 148.4, 158.9 and $188.5 \mathrm{ppm}$ (Ar-C and CO); MS (EI): $\mathrm{m} / z$ (\%) $466\left([\mathrm{M}+1]^{+}, 40.45\right)$, $465\left(\mathrm{M}^{+}, 100\right)$; HRMS (EI): $m / z$ calcd. for $\mathrm{C}_{23} \mathrm{H}_{13}{ }^{35} \mathrm{Cl}_{2} \mathrm{~N}_{3} \mathrm{O}_{4}\left(\mathrm{M}^{+}\right) 465.0277$, found 465.0277 .

6-(4-Bromobenzoyl)-2-(4-chloro-3-nitrophenyl)-4-(4-nitrophenyl)pyridazin-3-(2H)-one (3g). Yellow crystals, yield: $\left(2.5\right.$ g, 91\%), m.p.: 236-237 ${ }^{\circ} \mathrm{C}$; IR (KBr): $v / \mathrm{cm}^{-1} 1676,1648$ (2CO); ${ }^{1} \mathrm{H}-\mathrm{NMR}$ (DMSO- $\left.d_{6}\right): \delta=7.80$ (d, $\left.J=7.6 \mathrm{~Hz}, 2 \mathrm{H}, \mathrm{Ar}-\mathrm{H}\right), 7.99-8.10(\mathrm{~m}, 4 \mathrm{H}, \mathrm{Ar}-\mathrm{H}), 8.21$ (d, $\left.J=7.6 \mathrm{~Hz}, 2 \mathrm{H}, \mathrm{Ar}-\mathrm{H}\right)$, 8.29 (s, 1H, pyridazine H5), $8.38(\mathrm{~d}, J=7.6 \mathrm{~Hz}, 2 \mathrm{H}, \mathrm{Ar}-\mathrm{H})$ and $8.56 \mathrm{ppm}(\mathrm{s}, 1 \mathrm{H}, \mathrm{Ar}-\mathrm{H}) ;{ }^{13} \mathrm{C}-\mathrm{NMR}$ (DMSO- $\left.d_{6}\right): \delta=123.6,123.8,125.7,128.2,129.3,130.7,131.5,132.0,132.7,132.8,134.7,138.5$, 139.9, 141.1, 143.4, 147.7, 148.8, 158.9 and $188.4 \mathrm{ppm}$ (Ar-C and CO); MS (EI): $m / z(\%) 556\left([\mathrm{M}+2]^{+}\right.$, 87.88), $555\left([\mathrm{M}+1]^{+}, 41.10\right), 554\left(\mathrm{M}^{+}, 65.05\right)$; HRMS (EI): $m / z$ calcd. for $\mathrm{C}_{23} \mathrm{H}_{12}{ }^{79} \mathrm{BrClN}_{4} \mathrm{O}_{6}\left(\mathrm{M}^{+}\right)$ 553.9623 , found 553.9625 .

6-(4-Bromobenzoyl)-2-(4-chloro-3-nitrophenyl)-4-(2-nitrophenyl)pyridazin-3-(2H)-one (3h). Buff crystals, yield: $(2.13 \mathrm{~g}, 77 \%)$, m.p.: $218-219{ }^{\circ} \mathrm{C}$; IR (KBr): $v / \mathrm{cm}^{-1} 1679,1659$ (2CO); ${ }^{1} \mathrm{H}-\mathrm{NMR}$ (DMSO- $\left.d_{6}\right): \delta=7.77-7.85(\mathrm{~m}, 4 \mathrm{H}, \mathrm{Ar}-\mathrm{H}), 7.94-8.01(\mathrm{~m}, 3 \mathrm{H}, \mathrm{Ar}-\mathrm{H}), 8.07$ (d, $\left.J=8.8 \mathrm{~Hz}, 2 \mathrm{H}, \mathrm{Ar}-\mathrm{H}\right)$, $8.21(\mathrm{~d}, J=8.0 \mathrm{~Hz}, 1 \mathrm{H}, \mathrm{Ar}-\mathrm{H}), 8.26(\mathrm{~s}, 1 \mathrm{H}$, pyridazine $\mathrm{H} 5)$ and $8.45 \mathrm{ppm}(\mathrm{d}, J=2.0 \mathrm{~Hz}, 1 \mathrm{H}, \mathrm{Ar}-\mathrm{H})$; ${ }^{13} \mathrm{C}-\mathrm{NMR}\left(\mathrm{DMSO}-d_{6}\right): \delta=123.5,124.9,125.8,128.2,128.4,128.7,131.3,131.8,132.0,132.8,133.0$, 133.1, 134.5, 134.9, 140.5, 140.9, 143.5, 147.6, 148.7, 158.3 and 188.4 ppm (Ar-C and CO); MS (EI): $m / z(\%) 556\left([\mathrm{M}+2]^{+}, 8.85\right), 555\left([\mathrm{M}+1]^{+}, 3.44\right), 554\left(\mathrm{M}^{+}, 5.15\right)$; HRMS (EI): $m / z$ calcd. for $\mathrm{C}_{23} \mathrm{H}_{12}{ }^{79} \mathrm{BrClN}_{4} \mathrm{O}_{6}\left(\mathrm{M}^{+}\right)$553.9623, found 553.9624.

6-(4-Bromobenzoyl)-2-(4-chloro-3-nitrophenyl)-3-oxo-2,3-dihydropyridazine-4-carbonitrile

(3i). Brown crystals, yield: $\left(1.7\right.$ g, 75\%), m.p.: above $300{ }^{\circ} \mathrm{C}$; IR (KBr): $v / \mathrm{cm}^{-1} 2220(\mathrm{CN}), 1688,1654(2 \mathrm{CO})$; ${ }^{1} \mathrm{H}-\mathrm{NMR}$ (DMSO- $\left.d_{6}\right): \delta=7.79(\mathrm{~d}, J=8.0 \mathrm{~Hz}, 2 \mathrm{H}, \mathrm{Ar}-\mathrm{H}), 7.98(\mathrm{~d}, J=8.0 \mathrm{~Hz}, 2 \mathrm{H}, \mathrm{Ar}-\mathrm{H}), 8.01-8.04$ (m, $2 \mathrm{H}, \mathrm{Ar}-\mathrm{H}), 8.47$ (s, $1 \mathrm{H}$, pyridazine $\mathrm{H} 5)$ and $8.83 \mathrm{ppm}(\mathrm{s}, 1 \mathrm{H}, \mathrm{Ar}-\mathrm{H}) ;{ }^{13} \mathrm{C}-\mathrm{NMR}$ (DMSO- $\left.d_{6}\right): \delta=113.8$, $116.1,123.6,126.5,128.6,131.5,132.1,133.0,133.1,134.0,139.5,139.9,142.3,147.5,156.5$ and 187.5 ppm (Ar-C, CN and CO); MS (EI): $m / z(\%) 460\left([\mathrm{M}+2]^{+}, 22.58\right), 459\left([\mathrm{M}+1]^{+}, 7.04\right), 458$ $\left(\mathrm{M}^{+}, 16.81\right)$; HRMS (EI): $m / z$ calcd. for $\mathrm{C}_{18} \mathrm{H}_{8}{ }^{79} \mathrm{BrClN}_{4} \mathrm{O}_{4}\left(\mathrm{M}^{+}\right)$457.9411, found 457.9411 . 
6-(4-Chlorobenzoyl)-2-(4-chlorophenyl)-3-oxo-2,3-dihydropyridazine-4-carbonitrile (3j). Brown crystals, yield: $(1.5 \mathrm{~g}, 81 \%)$, m.p.: above $300{ }^{\circ} \mathrm{C}$; IR (KBr): v/ $\mathrm{cm}^{-1} 2218(\mathrm{CN}), 1689,1662(2 \mathrm{CO})$; ${ }^{1} \mathrm{H}-\mathrm{NMR}$ (DMSO- $\left.d_{6}\right): \delta=7.62-7.70(\mathrm{~m}, 4 \mathrm{H}, \mathrm{Ar}-\mathrm{H}), 7.96(\mathrm{~d}, J=8.4 \mathrm{~Hz}, 2 \mathrm{H}, \mathrm{Ar}-\mathrm{H}), 8.00(\mathrm{~d}, J=8.4 \mathrm{~Hz}$, $2 \mathrm{H}, \mathrm{Ar}-\mathrm{H})$ and $8.77 \mathrm{ppm}\left(\mathrm{s}, 1 \mathrm{H}\right.$, pyridazine H5); MS (EI): $\mathrm{m} / z(\%) 370\left([\mathrm{M}+1]^{+}, 35.22\right), 369\left(\mathrm{M}^{+}\right.$, 59.38); HRMS (EI): $m / z$ calcd. for $\mathrm{C}_{18} \mathrm{H}_{9} \mathrm{Cl}_{2} \mathrm{~N}_{3} \mathrm{O}_{2}\left(\mathrm{M}^{+}\right)$369.0066, found 369.0063 .

6-Benzoyl-2-(4-methoxyphenyl)-3-oxo-2,3-dihydropyridazine-4-carbonitrile (3k). Brown crystals, yield: $\left(1.4\right.$ g, 80\%), m.p.: 164-165 ${ }^{\circ} \mathrm{C}$; IR (KBr): v/cm ${ }^{-1} 2219(\mathrm{CN}), 1695,1659$ (2CO); ${ }^{1} \mathrm{H}-\mathrm{NMR}$ $\left(\mathrm{DMSO}-d_{6}\right): \delta=3.85\left(\mathrm{~s}, 3 \mathrm{H}, \mathrm{OCH}_{3}\right), 7.07$ (d, $\left.J=8.8 \mathrm{~Hz}, 2 \mathrm{H}, \mathrm{Ar}-\mathrm{H}\right), 7.35-7.65(\mathrm{~m}, 5 \mathrm{H}, \mathrm{Ar}-\mathrm{H}), 8.04(\mathrm{~d}$, $J=8.4 \mathrm{~Hz}, 2 \mathrm{H}, \mathrm{Ar}-\mathrm{H})$ and $8.75 \mathrm{ppm}\left(\mathrm{s}, 1 \mathrm{H}\right.$, pyridazine H5); MS (EI): $m / z(\%) 332\left([\mathrm{M}+1]^{+}, 16.85\right)$, $331\left(\mathrm{M}^{+}, 68.11\right)$; HRMS (EI): $m / z$ calcd. for $\mathrm{C}_{19} \mathrm{H}_{13} \mathrm{~N}_{3} \mathrm{O}_{3}\left(\mathrm{M}^{+}\right)$331.0951, found 331.0949.

6-Acety-4-(4-nitrophenyl)l-2-phenylpyridazin-3-(2H)-one (31). Yellow crystals, yield: (1.5 g, 89\%), m.p.: $182-183{ }^{\circ} \mathrm{C}$; IR (KBr): $v / \mathrm{cm}^{-1} 1689,1672(2 \mathrm{CO}) ;{ }^{1} \mathrm{H}-\mathrm{NMR}\left(\mathrm{DMSO}-d_{6}\right): \delta=2.51\left(\mathrm{~s}, 3 \mathrm{H}, \mathrm{CH}_{3}\right)$, $7.51(\mathrm{t}, J=7.8 \mathrm{~Hz}, 1 \mathrm{H}, \mathrm{Ar}-\mathrm{H}), 7.58(\mathrm{t}, J=7.8 \mathrm{~Hz}, 2 \mathrm{H}, \mathrm{Ar}-\mathrm{H}), 7.70$ (d, $J=7.8 \mathrm{~Hz}, 2 \mathrm{H}, \mathrm{Ar}-\mathrm{H}), 8.11$ (s, $1 \mathrm{H}$, pyridazine $\mathrm{H} 5), 8.14(\mathrm{~d}, J=8.4 \mathrm{~Hz}, 2 \mathrm{H}, \mathrm{Ar}-\mathrm{H})$ and $8.31 \mathrm{ppm}(\mathrm{d}, J=8.4 \mathrm{~Hz}, 2 \mathrm{H}, \mathrm{Ar}-\mathrm{H}) ;{ }^{13} \mathrm{C}-\mathrm{NMR}$ (DMSO- $\left.d_{6}\right): \delta=25.1\left(\mathrm{CH}_{3}\right), 123.8,126.4,126.8,129.25,129.30,130.7,137.5,140.4,142.0,142.5$, 148.3, 159.1 and $195.1 \mathrm{ppm}$ (Ar-C and CO); MS (EI): $m / z(\%) 336\left([\mathrm{M}+1]^{+}, 25.08\right), 335\left(\mathrm{M}^{+}, 100\right)$; HRMS (EI): $m / z$ calcd. for $\mathrm{C}_{18} \mathrm{H}_{13} \mathrm{~N}_{3} \mathrm{O}_{4}\left(\mathrm{M}^{+}\right)$335.0900, found 335.0901. Crystal Data, $\mathrm{C}_{18} \mathrm{H}_{13} \mathrm{~N}_{3} \mathrm{O}_{4}$, $\mathrm{M}=335.32$, monoclinic, $\mathrm{a}=25.68(3) \AA, \mathrm{b}=3.848(4) \AA, \mathrm{c}=32.02(3) \AA, \mathrm{V}=3164(5) \AA^{3}, \alpha=\gamma=90^{\circ}$, $\beta=90.62(2)^{\circ}$, space group: $C 2 / \mathrm{c}(\# 15), Z=8, D_{\text {calc }}=1.408 \mathrm{~g} \cdot \mathrm{cm}^{-3}$, No. of reflection measured 2806,2 $\theta_{\text {max }}=50.1^{\circ}, \mathrm{R} 1=0.0818[39]$.

(E)-6-(3-(Dimethylamino)acryloyl)-4-(4-nitrophenyl)l-2-phenylpyridazin-3-(2H)-one (4). Mixture of 31 (1.68 g, $5 \mathrm{mmol}$ ), $\mathrm{N}, \mathrm{N}$-dimethylformamide dimethylacetal (DMF-DMA) (0.6 g, $5 \mathrm{mmol}$ ) in dioxane $(20 \mathrm{~mL})$ were stirred at reflux for $5 \mathrm{~h}$. The separated solid product obtained on standing at room temperature was collected by filtration, washed by $\mathrm{EtOH}$ and recrystallized from dioxane to afford the corresponding enamines 4 as orange crystal. Yellow crystals, yield: $\left(1.70\right.$ g, 90\%), m.p.: $214-215^{\circ} \mathrm{C}$; IR (KBr): $v / \mathrm{cm}^{-1} 1678,1644$ (2CO); ${ }^{1} \mathrm{H}-\mathrm{NMR}$ (DMSO-d $\left.)_{6}\right): \delta=2.84\left(\mathrm{~s}, 3 \mathrm{H}, \mathrm{CH}_{3}\right), 3.15\left(\mathrm{~s}, 3 \mathrm{H}, \mathrm{CH}_{3}\right)$, $5.88(\mathrm{~d}, J=12 \mathrm{~Hz}, 1 \mathrm{H}$, olefinic $\mathrm{CH}=\mathrm{CH}), 7.49(\mathrm{t}, J=7.8 \mathrm{~Hz}, 1 \mathrm{H}, \mathrm{Ar}-\mathrm{H}), 7.56(\mathrm{t}, J=7.8 \mathrm{~Hz}, 2 \mathrm{H}, \mathrm{Ar}-\mathrm{H})$, $7.67(\mathrm{~d}, J=7.8 \mathrm{~Hz}, 2 \mathrm{H}, \mathrm{Ar}-\mathrm{H}), 7.81(\mathrm{~d}, J=12 \mathrm{~Hz}, 1 \mathrm{H}$, olefinic $\mathrm{CH}=\mathrm{CH}), 8.13(\mathrm{~d}, J=8.4 \mathrm{~Hz}, 2 \mathrm{H}, \mathrm{Ar}-\mathrm{H})$, $8.15(\mathrm{~s}, 1 \mathrm{H}$, pyridazine $\mathrm{H} 5)$ and $8.30 \mathrm{ppm}(\mathrm{d}, J=8.4 \mathrm{~Hz}, 2 \mathrm{H}, \mathrm{Ar}-\mathrm{H}) ;{ }^{13} \mathrm{C}-\mathrm{NMR}\left(\mathrm{DMSO}-d_{6}\right): \delta=37.6$ $\left(\mathrm{CH}_{3}\right), 45.1\left(\mathrm{CH}_{3}\right), 88.6,123.7,126.6,127.9,128.9,129.30,130.6,137.3,140.9,142.3,144.3,148.1$, 155.1, 159.1 and 180.7 ppm (Ar-C and CO); MS (EI): $m / z(\%) 391\left([\mathrm{M}+1]^{+}, 19.75\right), 335\left(\mathrm{M}^{+}, 85.07\right)$; HRMS (EI): $m / z$ calcd. for $\mathrm{C}_{21} \mathrm{H}_{18} \mathrm{~N}_{4} \mathrm{O}_{4}\left(\mathrm{M}^{+}\right)$390.1322, found 390.1322. Anal. calcd. for $\mathrm{C}_{21} \mathrm{H}_{18} \mathrm{~N}_{4} \mathrm{O}_{4}$ (390.40): C, 64.61; H, 4.65; N, 14.35. Found C, 64.80; H, 4.77; N, 14.53.

\subsubsection{General Procedure for the Synthesis of Azolopyrimidines 5-7}

Independent mixtures of $4(0.78 \mathrm{~g}, 2 \mathrm{mmol})$ and the appropriate heteroaromatic amine $(2 \mathrm{mmol})$ in pyridine $(20 \mathrm{~mL})$ were stirred at reflux for $24 \mathrm{~h}$. The reaction mixtures were cooled to room temperature and poured into ice cold water then acidified with hydrochloric acid $(2 \mathrm{~N})$, forming solids 
that were collected by filtration and washed with water then $\mathrm{MeOH}$ and recrystallized from the appropriate solvent.

4-(4-Nitrophenyl)-2-phenyl-6-(1,2,4-triazolo[1,5-a]pyrimidin-7-yl)-(2H)-pyridazin-3-one (5). Pall yellow crystals, yield: $(0.7 \mathrm{~g}, 76 \%)$, m.p.: $149-150{ }^{\circ} \mathrm{C}$; IR $(\mathrm{KBr}): v / \mathrm{cm}^{-1} 1668$ (CO); ${ }^{1} \mathrm{H}-\mathrm{NMR}$ $\left(\mathrm{DMSO}-d_{6}\right): \delta=7.54(\mathrm{t}, J=8.0 \mathrm{~Hz}, 1 \mathrm{H}, \mathrm{Ar}-\mathrm{H}), 7.61(\mathrm{t}, J=8.0 \mathrm{~Hz}, 2 \mathrm{H}, \mathrm{Ar}-\mathrm{H}), 7.79$ (d, $J=8.0 \mathrm{~Hz}, 2 \mathrm{H}$, Ar-H), 7.98 (d, $J=5.4 \mathrm{~Hz}, 1 \mathrm{H}$, pyrimidine H6), 8.25 (d, $J=8.0 \mathrm{~Hz}, 2 \mathrm{H}, \mathrm{Ar}-\mathrm{H}), 8.38$ (d, $J=8.0 \mathrm{~Hz}$, $2 \mathrm{H}, \mathrm{Ar}-\mathrm{H}), 8.66(\mathrm{~s}, 1 \mathrm{H}$, pyridazine $\mathrm{H} 5), 8.80(\mathrm{~s}, 1 \mathrm{H}$, triazole $\mathrm{H} 2)$ and $9.48 \mathrm{ppm}(\mathrm{d}, J=5.4 \mathrm{~Hz}, 1 \mathrm{H}$, pyrimidine H5); MS (EI): $m / z(\%) 461\left([\mathrm{M}+1]^{+}, 30.78\right), 460\left(\mathrm{M}^{+}, 100\right)$; HRMS (EI): $\mathrm{m} / z$ calcd. for $\mathrm{C}_{26} \mathrm{H}_{16} \mathrm{~N}_{6} \mathrm{O}_{3}\left(\mathrm{M}^{+}\right) 460.1278$, found 460.1278 .

4-(4-Nitrophenyl)-2-phenyl-6-(2-phenylpyrazolo[1,5-a]pyrimidin-7-yl)-(2H)-pyridazin-3-one

Yellow crystals, yield: $(0.77 \mathrm{~g}, 80 \%)$, m.p.: 285-286 ${ }^{\circ} \mathrm{C}$; IR (KBr): v/cm ${ }^{-1} 1671$ (CO); ${ }^{1} \mathrm{H}-\mathrm{NMR}$ (DMSO- $\left.d_{6}\right): \delta=7.43-7.45(\mathrm{~m}, 2 \mathrm{H}, \mathrm{Ar}-\mathrm{H}), 7.49(\mathrm{~d}, J=4.8 \mathrm{~Hz}, 1 \mathrm{H}$, pyrimidine H6), 7.51-7.54 (m, 3H, Ar-H), 7.59 (t, $J=7.8 \mathrm{~Hz}, 2 \mathrm{H}, \mathrm{Ar}-\mathrm{H}), 7.80$ (d, $J=7.8 \mathrm{~Hz}, 2 \mathrm{H}, \mathrm{Ar}-\mathrm{H}), 8.05$ (d, $J=7.8 \mathrm{~Hz}, 2 \mathrm{H}, \mathrm{Ar}-\mathrm{H})$, $8.23(\mathrm{~d}, J=8.4 \mathrm{~Hz}, 2 \mathrm{H}, \mathrm{Ar}-\mathrm{H}), 8.40(\mathrm{~d}, J=8.4 \mathrm{~Hz}, 2 \mathrm{H}, \mathrm{Ar}-\mathrm{H}), 8.67$ (d, $J=4.8 \mathrm{~Hz}, 1 \mathrm{H}$, pyrimidine H5) and $8.96 \mathrm{ppm}(\mathrm{s}, 1 \mathrm{H}$, pyridazine $\mathrm{H} 5) ;{ }^{13} \mathrm{C}-\mathrm{NMR}$ (DMSO- $\left.d_{6}\right): \delta=93.9,107.9,123.5,126.1,126.2$, 128.7, 128.8, 129.0, 129.3, 130.1, 130.9, 132.1, 136.1, 138.0, 139.9, 140.2, 141.5, 147.9, 149.9, 150.6, 155.0 and $158.3 \mathrm{ppm}$ (Ar-C and CO); MS (EI): $m / z(\%) 487\left([\mathrm{M}+1]^{+}, 35.02\right), 486\left(\mathrm{M}^{+}, 100\right)$; HRMS (EI): $m / z$ calcd. for $\mathrm{C}_{28} \mathrm{H}_{18} \mathrm{~N}_{6} \mathrm{O}_{3}\left(\mathrm{M}^{+}\right)$486.1434, found 486.1432. Anal. calcd. for $\mathrm{C}_{28} \mathrm{H}_{18} \mathrm{~N}_{6} \mathrm{O}_{3}$ (486.49): C, 69.13; H, 3.73; N, 17.27. Found C, 69.27; H, 3.65; N, 17.39.

6-(Benzo[4,5]imidazo[1,2-a]pyrimidine-4-yl)-4-(4-nitrophenyl)-2-phenyl-(2H)-pyridazin-3-one

Yellow crystals, yield: (0.6 g, 73\%), m.p.: above $300{ }^{\circ} \mathrm{C}$; IR (KBr): v/cm ${ }^{-1} 1669$ (CO); ${ }^{1} \mathrm{H}-\mathrm{NMR}$ $\left(\mathrm{DMSO}-d_{6}\right): \delta=7.45(\mathrm{t}, J=7.8 \mathrm{~Hz}, 1 \mathrm{H}, \mathrm{Ar}-\mathrm{H}), 7.54(\mathrm{t}, J=7.8 \mathrm{~Hz}, 1 \mathrm{H}, \mathrm{Ar}-\mathrm{H}), 7.57-762(\mathrm{~m}, 3 \mathrm{H}, \mathrm{Ar}-\mathrm{H})$, $7.74(\mathrm{~d}, J=5.4 \mathrm{~Hz}, 1 \mathrm{H}$, pyrimidine H6), $7.80(\mathrm{~d}, J=8.4 \mathrm{~Hz}, 2 \mathrm{H}, \mathrm{Ar}-\mathrm{H}), 7.88(\mathrm{~d}, J=7.8 \mathrm{~Hz}, 1 \mathrm{H}, \operatorname{Ar}-\mathrm{H})$, 8.24 (d, $J=9.0 \mathrm{~Hz}, 2 \mathrm{H}, \mathrm{Ar}-\mathrm{H}), 8.28$ (d, $J=7.8 \mathrm{~Hz}, 1 \mathrm{H}, \mathrm{Ar}-\mathrm{H}), 8.34$ (d, $J=9.0 \mathrm{~Hz}, 2 \mathrm{H}, \mathrm{Ar}-\mathrm{H}), 8.69$ (s, $1 \mathrm{H}$, pyridazine $\mathrm{H} 5)$ and $9.48 \mathrm{ppm}(\mathrm{d}, J=5.4 \mathrm{~Hz}, 1 \mathrm{H}$, pyrimidine $\mathrm{H} 5) ;{ }^{13} \mathrm{C}-\mathrm{NMR}$ (DMSO- $\left.d_{6}\right)$ : $\delta=102.9,112.9,119.5,119.9,122.5,123.7,126.4,126.7,127.8,129.0,129.2,130.7,136.6,138.3$, 140.7, 142.4, 142.7, 145.2, 148.7, 150.2, 157.0 and 159.1 ppm (Ar-C and CO); MS (EI): $m / z(\%) 412$ $\left([\mathrm{M}+1]^{+}, 26.11\right), 411\left(\mathrm{M}^{+}, 100\right)$; $\operatorname{HRMS}(\mathrm{EI}): \mathrm{m} / z$ calcd. for $\mathrm{C}_{21} \mathrm{H}_{13} \mathrm{~N}_{7} \mathrm{O}_{3}\left(\mathrm{M}^{+}\right)$411.1074, found 411.1074 .

\subsubsection{General Procedure for the Preparation of Compounds 16-20}

Independent mixtures of $\mathbf{8 a}(1.03 \mathrm{~g}, 2.5 \mathrm{mmol})$, cyanoacetylindoles 11,12, cyanoacetamides 13-15 ( $2.5 \mathrm{mmol})$, and ammonium acetate $(2 \mathrm{~g})$ in acetic acid $(20 \mathrm{~mL})$ were stirred at reflux for $1-2 \mathrm{~h}$. (progress of the reactions monitored by using TLC with 1:1 ethyl acetate/petroleum ether as eluent). The mixtures were cooled to room temperature. The formed solids were collected by filtration and crystallized from the indicated solvents to give $\mathbf{1 6}-\mathbf{2 0}$ as pure products.

[2-Amino-6-(4-bromophenyl)-5-(4-chloro-3-nitrophenylazo)pyridin-3-yl](1-methyl-1H-indol-3-yl)methanone (16). Recrystallized from EtOH/DMF mixture (1:1) as pale brown crystals, yield: (1.10 g, 75\%), m.p.: $273-274{ }^{\circ} \mathrm{C}$; IR (KBr): $v / \mathrm{cm}^{-1} 3329,3279\left(\mathrm{NH}_{2}\right), 1635(\mathrm{CO}) ;{ }^{1} \mathrm{H}-\mathrm{NMR}\left(\mathrm{DMSO}-d_{6}\right): \delta=3.89$ 
$\left(\mathrm{s}, 3 \mathrm{H}, \mathrm{CH}_{3}\right), 7.32$ (t, $\left.J=8.0 \mathrm{~Hz}, 1 \mathrm{H}, \mathrm{Ar}-\mathrm{H}\right), 7.37$ (t, $\left.J=8.0 \mathrm{~Hz}, 1 \mathrm{H}, \mathrm{Ar}-\mathrm{H}\right), 7.62(\mathrm{~d}, J=8.0 \mathrm{~Hz}, 1 \mathrm{H}$, $\operatorname{Ar}-\mathrm{H}), 7.74(\mathrm{~d}, J=8.4 \mathrm{~Hz}, 2 \mathrm{H}, \mathrm{Ar}-\mathrm{H}), 7.82(\mathrm{~d}, J=8.4 \mathrm{~Hz}, 2 \mathrm{H}, \mathrm{Ar}-\mathrm{H}), 7.87$ (s, 2H, NH ), $7.88-7.93$ (m, 2H, Ar-H), 8.13 (s, 1H, indole H2), 8.24 (d, $J=8.0 \mathrm{~Hz}, 1 \mathrm{H}, \mathrm{Ar}-\mathrm{H}), 8.30(\mathrm{~s}, 1 \mathrm{H}, \mathrm{Ar}-\mathrm{H})$ and 8.39 ppm (s, $1 \mathrm{H}$, pyridine $\mathrm{H} 4) ;{ }^{13} \mathrm{C}-\mathrm{NMR}$ (DMSO- $d_{6}$ at $80{ }^{\circ} \mathrm{C}$ ): $\delta=33.7\left(\mathrm{CH}_{3}\right), 111.2$ (pyridine $\mathrm{C} 3$ ), 115.4, 117.3, 119.5, 122.1, 122.8, 123.6, 123.9, 126.2, 127.0, 127.2, 127.5, 131.0, 133.2, 133.4, 137.1, 138.2, 139.3, 145.0, 148.8, 152.0, 159.9, 160.2 and $189.0 \mathrm{ppm}$ (Ar-C and CO); MS (EI): $m / z(\%) 590\left([\mathrm{M}+2]^{+}\right.$, 37.92), $589\left([\mathrm{M}+1]^{+}, 36.11\right), 588\left(\mathrm{M}^{+}, 26.44\right)$; HRMS (EI): $m / z$ Calcd. for $\mathrm{C}_{27} \mathrm{H}_{18}{ }^{79} \mathrm{Br}^{35} \mathrm{ClN}_{6} \mathrm{O}_{3}\left(\mathrm{M}^{+}\right)$ 588.0306, found 588.0306. Anal. calcd. for $\mathrm{C}_{27} \mathrm{H}_{18} \mathrm{BrClN}_{6} \mathrm{O}_{3}$ (589.84): C, 54.98; H, 3.08; N, 14.25 . Found C, 54.83; H, 3.17; N, 14.11.

[2-Amino-6-(4-bromophenyl)-5-(4-chloro-3-nitrophenylazo)pyridin-3-yl](2-methyl-1H-indol-3-yl)methanone (17). Recrystallized from EtOH/DMF mixture (1:1) as pale brown crystals, yield: (1.16 g, 79\%), m.p.: $292-293{ }^{\circ} \mathrm{C}$; IR (KBr): $v / \mathrm{cm}^{-1} 3345,3267,3168\left(\mathrm{NH}_{2}\right.$ and $\left.\mathrm{NH}\right), 1631\left((\mathrm{CO})\right.$; ${ }^{1} \mathrm{H}-\mathrm{NMR}$ $\left(\mathrm{DMSO}-d_{6}\right): \delta=2.48\left(\mathrm{~s}, 3 \mathrm{H}, \mathrm{CH}_{3}\right), 7.06(\mathrm{t}, J=8.0 \mathrm{~Hz}, 1 \mathrm{H}, \mathrm{Ar}-\mathrm{H}), 7.15(\mathrm{t}, J=8.0 \mathrm{~Hz}, 1 \mathrm{H}, \mathrm{Ar}-\mathrm{H}), 7.42$ (d, $J=8.0 \mathrm{~Hz}, 1 \mathrm{H}, \mathrm{Ar}-\mathrm{H}), 7.53$ (d, $J=8.0 \mathrm{~Hz}, 1 \mathrm{H}, \mathrm{Ar}-\mathrm{H}), 7.73(\mathrm{~d}, J=8.4 \mathrm{~Hz}, 2 \mathrm{H}, \mathrm{Ar}-\mathrm{H}), 7.80-7.88$ (m, 4H, Ar-H),7.94 (s, 2H, NH $), 8.19(\mathrm{~d}, J=2.4 \mathrm{~Hz}, 1 \mathrm{H}, \mathrm{Ar}-\mathrm{H}), 8.26(\mathrm{~s}, 1 \mathrm{H}$, pyridine H4) and $12.07 \mathrm{ppm}$ $(\mathrm{s}, 1 \mathrm{H}, \mathrm{NH}) ;{ }^{13} \mathrm{C}-\mathrm{NMR}\left(\mathrm{DMSO}-d_{6}\right): \delta=14.4\left(\mathrm{CH}_{3}\right), 111.5$ (pyridine $\mathrm{C} 3$ ), 112.7, 117.1, 119.4, 119.9, 121.3, 122.1, 123.2, 125.7, 126.3, 127.1, 127.8, 130.6, 132.7, 133.2, 135.0, 136.1, 136.4, 144.4, 148.0, 151.1, 159.5, 160.0 and $190.2 \mathrm{ppm}$ (Ar-C and CO); MS (EI): $m / z(\%) 590\left([\mathrm{M}+2]^{+}, 28.79\right), 589$ $\left([\mathrm{M}+1]^{+}, 26.98\right), 588\left(\mathrm{M}^{+}, 21.03\right)$; HRMS (EI): $m / z$ Calcd. for $\mathrm{C}_{27} \mathrm{H}_{18}{ }^{79} \mathrm{BrClN}_{6} \mathrm{O}_{3}\left(\mathrm{M}^{+}\right)$588.0306, found 588.0307 .

2-Amino-6-(4-bromophenyl)-N-(4-chloro-3-nitrophenyl)-5-(4-chloro-3-nitrophenylazo)nicotin-amide (18). Recrystallized from dioxane as orange crystals, yield: $(1.27 \mathrm{~g}, 81 \%)$, m.p.: $299-300{ }^{\circ} \mathrm{C}$; IR $(\mathrm{KBr})$ : $v / \mathrm{cm}^{-1}$ 3371, 3266, $3170\left(\mathrm{NH}_{2}\right.$ and $\left.\mathrm{NH}\right), 1658(\mathrm{CO}) ;{ }^{1} \mathrm{H}-\mathrm{NMR}\left(\mathrm{DMSO}-d_{6}\right): \delta=7.69(\mathrm{~d}, J=8.4 \mathrm{~Hz}$, 2H, Ar-H), 7.74-7.76 (m, 3H, Ar-H), 7.91-7.99 (m, 3H, Ar-H), 8.04 (s, 2H, NH 2$), 8.28$ (d, J=2.4 Hz, $1 \mathrm{H}, \mathrm{Ar}-\mathrm{H}), 8.50$ (d, $J=2.4 \mathrm{~Hz}, 1 \mathrm{H}, \mathrm{Ar}-\mathrm{H}), 8.57$ (s, 1H, pyridine $\mathrm{H} 4)$ and $11.00 \mathrm{ppm}(\mathrm{s}, 1 \mathrm{H}, \mathrm{NH})$; ${ }^{13} \mathrm{C}-\mathrm{NMR}$ (DMSO- $d_{6}$ ): $\delta=110.2$ (pyridine C3), 117.0, 119.2, 119.3, 123.3, 125.4, 125.6, 126.0, 126.7, 130.5, 131.9, 133.0, 133.1, 135.9, 136.2, 138.7, 147.0, 148.0, 151.1, 159.9, 160.4 and 166.1 ppm (Ar-C and CO); MS (EI): $m / z(\%) 631\left([\mathrm{M}+2]^{+}, 66.14\right), 630\left([\mathrm{M}+1]^{+}, 100\right), 629\left(\mathrm{M}^{+}, 50.07\right)$; HRMS (EI): $m / z$ Calcd. for $\mathrm{C}_{24} \mathrm{H}_{14}{ }^{79} \mathrm{BrCl}_{2} \mathrm{~N}_{7} \mathrm{O}_{5}\left(\mathrm{M}^{+}\right)$628.9611, found 628.9611. Crystal Data, $\mathrm{C}_{52} \mathrm{H}_{36} \mathrm{Br}_{2} \mathrm{Cl}_{4} \mathrm{~N}_{14} \mathrm{O}_{12}$, $\mathrm{M}=1350.57$, triclinic, $\mathrm{a}=8.0796(5) \AA, \mathrm{b}=11.0560(7) \AA, \mathrm{c}=16.1457(11) \AA, \mathrm{V}=1336.71(15) \AA^{3}$, $\alpha=72.539(5)^{\circ}, \beta=76.338(5)^{\circ}, \gamma=86.790(6)^{\circ}$, space group: $\mathrm{P}-1, \mathrm{Z}=1, \mathrm{D}_{\text {calc }}=1.678 \mathrm{~g} \cdot \mathrm{cm}^{-3}$, No. of reflection measured $6079,2 \theta_{\max }=27.44^{\circ}, \mathrm{R} 1=0.0386$ [42].

N-(5-Acetyl-2-phenyl-2H-1,2,3-triazol-4-yl)-2-amino-6-(4-bromophenyl)-5-(4-chloro-3-nitrophenyl)nicotinamide (19). Recrystallized from dioxane as deep orange crystals, yield: (1.19 g. 72\%), m.p.: above $300{ }^{\circ} \mathrm{C}$; IR (KBr): v/cm ${ }^{-1} 3382,3351,3166\left(\mathrm{NH}_{2}\right.$ and $\left.\mathrm{NH}\right), 1689,1659$ ( (2CO); ${ }^{1} \mathrm{H}-\mathrm{NMR}$ $\left(\mathrm{DMSO}-d_{6}\right): \delta=2.66\left(\mathrm{~s}, 3 \mathrm{H}, \mathrm{CH}_{3}\right), 7.54(\mathrm{t}, J=7.6 \mathrm{~Hz}, 1 \mathrm{H}, \mathrm{Ar}-\mathrm{H}), 7.65(\mathrm{t}, J=7.6 \mathrm{~Hz}, 2 \mathrm{H}, \mathrm{Ar}-\mathrm{H}), 7.72$ (d, $J=8.4 \mathrm{~Hz}, 2 \mathrm{H}, \mathrm{Ar}-\mathrm{H}), 7.79-7.82(\mathrm{~m}, 2 \mathrm{H}, \mathrm{Ar}-\mathrm{H}), 7.99$ (s, 2H, NH$), 8.07-8.10$ (m, 4H, Ar-H), 9.30 $(\mathrm{s}, 1 \mathrm{H}, \mathrm{Ar}-\mathrm{H}), 8.74(\mathrm{~s}, 1 \mathrm{H}$, pyridine $\mathrm{H} 4)$ and $11.21 \mathrm{ppm}(\mathrm{s}, 1 \mathrm{H}, \mathrm{NH})$; MS (EI): $m / z(\%) 661\left([\mathrm{M}+2]^{+}\right.$, 
77.95), $660\left([\mathrm{M}+1]^{+}, 100\right), 659\left(\mathrm{M}^{+}, 56.01\right)$; HRMS (EI): $m / z$ Calcd. for $\mathrm{C}_{28} \mathrm{H}_{19}{ }^{79} \mathrm{BrClN}_{9} \mathrm{O}_{4}\left(\mathrm{M}^{+}\right)$ 659.0426, found 659.0428 .

6-(4-Bromobenzoyl)-2-(4-chloro-3-nitrophenyl)-3-imino-2,3-dihydropyridazine-4-carboxylic acid (3-cyano-4-phenylthiophen-2-yl)amide (20a). Recrystallized from EtOH/DMF mixture (1:3) as pale brown crystals, yield: (1.27 g, 77\%), m.p.: $270-271{ }^{\circ} \mathrm{C}$; IR (KBr): $v / \mathrm{cm}^{-1} 3426,3346$ (2 NH), 2216 $(\mathrm{CN}), 1678,1659(\mathrm{CO}) ;{ }^{1} \mathrm{H}-\mathrm{NMR}$ (DMSO- $\left.d_{6}\right): \delta=7.16(\mathrm{~s}, 1 \mathrm{H}$, thiophene H5), $7.37(\mathrm{t}, J=8.0 \mathrm{~Hz}, 1 \mathrm{H}$, Ar-H), 7.47 (t, $J=8.0 \mathrm{~Hz}, 2 \mathrm{H}, \operatorname{Ar}-\mathrm{H}), 7.63$ (d, $J=8.0 \mathrm{~Hz}, 2 \mathrm{H}, \operatorname{Ar}-\mathrm{H}), 7.75$ (d, $J=8.8 \mathrm{~Hz}, 2 \mathrm{H}, \operatorname{Ar}-\mathrm{H})$, $7.95(\mathrm{~d}, J=8.8 \mathrm{~Hz}, 2 \mathrm{H}, \mathrm{Ar}-\mathrm{H}), 8.16-8.23$ (m, 2H, Ar-H), 8.73 (d, $J=2.0 \mathrm{~Hz}, 1 \mathrm{H}, \mathrm{Ar}-\mathrm{H}), 8.79$ (s, 1H, pyridazine H5), $9.85(\mathrm{~s}, 1 \mathrm{H}$, imine $\mathrm{NH})$ and $13.32 \mathrm{ppm}(\mathrm{s}, 1 \mathrm{H}$, amide $\mathrm{NH}) ;{ }^{13} \mathrm{C}-\mathrm{NMR}$ (DMSO- $\left.d_{6}\right)$ : $\delta=94.4$ (thiophene C-3), 114.9, 117.8, 125.3, 127.0, 127.7, 128.40, 128.42, 128.8, 130.9, 131.2, 131.7, 132.6, 132.8, 133.4, 134.51, 134.54, 136.8, 138.8, 146.3, 148.2, 155.0, 161.0, 163.4 and 187.4 ppm (Ar-C, CN and CO); MS (EI): $m / z(\%) 660\left([\mathrm{M}+2]^{+}, 7.95\right), 659\left([\mathrm{M}+1]^{+}, 2.73\right), 658\left(\mathrm{M}^{+}, 6.55\right)$; HRMS (EI): $m / z$ Calcd. for $\mathrm{C}_{29} \mathrm{H}_{16}{ }^{79} \mathrm{BrClN}_{6} \mathrm{O}_{4} \mathrm{~S}\left(\mathrm{M}^{+}\right)$657.9820, found 657.9820 .

6-(4-Bromobenzoyl)-2-(4-chloro-3-nitrophenyl)-3-imino-2,3-dihydropyridazine-4-carboxylic acid [4-(4-chlorophenyl)-3-cyanothiophen-2-yl]amide (20b). Recrystallized from EtOH/DMF mixture (1:3) as yellowish brown crystals, yield: $\left(1.26\right.$ g, 73\%), m.p.: $288-289{ }^{\circ} \mathrm{C}$; IR (KBr): $v / \mathrm{cm}^{-1} 3401,3349(2 \mathrm{NH})$, $2214(\mathrm{CN}), 1673,1654(\mathrm{CO}) ;{ }^{1} \mathrm{H}-\mathrm{NMR}\left(\mathrm{DMSO}-d_{6}\right): \delta=7.16(\mathrm{~s}, 1 \mathrm{H}$, thiophene H5), $7.53(\mathrm{~d}, J=8.8 \mathrm{~Hz}$, 2H, Ar-H), 7.65 (d, $J=8.4 \mathrm{~Hz}, 2 \mathrm{H}, \operatorname{Ar}-\mathrm{H}), 7.76$ (d, $J=8.8 \mathrm{~Hz}, 2 \mathrm{H}, \mathrm{Ar}-\mathrm{H}), 7.95$ (d, $J=8.4 \mathrm{~Hz}, 2 \mathrm{H}$, Ar-H), 8.17-8.19 (m, 2H, Ar-H), 8.71 (d, $J=2.0 \mathrm{~Hz}, 1 \mathrm{H}, \mathrm{Ar}-\mathrm{H}), 8.78$ (s, 1H, pyridazine H5), 9.84 (s, $1 \mathrm{H}$, imine $\mathrm{NH}$ ) and $13.25 \mathrm{ppm}(\mathrm{s}, 1 \mathrm{H}$, amide $\mathrm{NH}) ;{ }^{13} \mathrm{C}-\mathrm{NMR}$ (DMSO- $\left.d_{6}\right): \delta=94.1$ (thiophene C-3), 115.5, 117.6, 125.2, 128.4, 128.7, 128.8, 131.0, 131.2, 131.7, 132.4, 132.6, 132.8, 133.36, 133.39, 134.5, 135.4, 138.8, 146.3, 148.1, 155.0, 161.1, 163.7 and 187.5 ppm (Ar-C, CN and CO); MS (EI): $m / z(\%) 694\left([\mathrm{M}+2]^{+}, 11.84\right), 693\left([\mathrm{M}+1]^{+}, 5.12\right), 692\left(\mathrm{M}^{+}, 8.92\right)$; HRMS (EI): $m / z$ Calcd. for $\mathrm{C}_{29} \mathrm{H}_{15}{ }^{79} \mathrm{BrCl}_{2} \mathrm{~N}_{6} \mathrm{O}_{4} \mathrm{~S}\left(\mathrm{M}^{+}\right) 691.9430$, found 691.9432 .

3.2.4. General Procedure for the Preparation of Naphthyridine Derivatives 24a,b

Independent solutions of cyanoacetic acid $(0.425 \mathrm{~g}, 5 \mathrm{mmol})$ in $\mathrm{Ac}_{2} \mathrm{O}(10 \mathrm{~mL})$ was heated at $100^{\circ} \mathrm{C}$ for $5 \mathrm{~min}$. then compounds $\mathbf{1 0 a}, \mathbf{b}(5 \mathrm{mmol})$ were added and the reaction mixtures were heated for further $30 \mathrm{~min}$. at $100{ }^{\circ} \mathrm{C}$. Then the reaction mixtures were allowed to cool to room temperature and the formed crystalline solids were separated by filtration, washed with ethanol and recrystallized from the proper solvent.

7-(4-Bromophenyl)-6-(4-chloro-3-nitrophenylazo)-2-oxo-4-phenyl-1,2-dihydro[1,8]naphthyridine-3carbonitrile (24a).Recrystallized from EtOH/dioxane mixture (1:1) as pale brown crystals, yield: (2.54 g, 80\%), m.p.: above $300{ }^{\circ} \mathrm{C}$; IR (KBr): $v / \mathrm{cm}^{-1} 3397(\mathrm{NH}), 2220(\mathrm{CN}), 1673(\mathrm{CO}) ;{ }^{1} \mathrm{H}-\mathrm{NMR}$ (DMSO- $\left.d_{6}\right)$ : $\delta=7.51-7.53(\mathrm{~m}, 2 \mathrm{H}, \mathrm{Ar}-\mathrm{H}), 7.62-7.63(\mathrm{~m}, 3 \mathrm{H}, \mathrm{Ar}-\mathrm{H}), 7.71(\mathrm{~d}, J=8.4 \mathrm{~Hz}, 2 \mathrm{H}, \mathrm{Ar}-\mathrm{H}), 7.77$ (d, J= 8.4 Hz, 2H, Ar-H), 7.80-7.89 (m, 3H, $2 \mathrm{Ar}-\mathrm{H}$ and pyridine $\mathrm{H} 4), 8.18$ (d, $J=2.4 \mathrm{~Hz}, 1 \mathrm{H}, \mathrm{Ar}-\mathrm{H})$ and $13.33 \mathrm{ppm}$ $(\mathrm{s}, 1 \mathrm{H}, \mathrm{NH}) ;{ }^{13} \mathrm{C}-\mathrm{NMR}\left(\mathrm{DMSO}-d_{6}\right): \delta=107.3,113.5,117.7,120.0,122.5,123.6,126.6,127.0,129.3$, $129.5,130.1,131.1,133.2,133.6,134.5,137.5,138.7,145.6,148.5,151.4,157.9,158.0$ and 160.7 ppm (Ar-C, CN and CO); MS (EI): $m / z(\%) 586\left([\mathrm{M}+2]^{+}, 61.03\right), 585\left([\mathrm{M}+1]^{+}, 84.11\right), 584\left(\mathrm{M}^{+}\right.$, 
47.05); HRMS (EI): $m / z$ calcd. for $\mathrm{C}_{27} \mathrm{H}_{14}{ }^{79} \mathrm{Br}^{35} \mathrm{ClN}_{6} \mathrm{O}_{3}\left(\mathrm{M}^{+}\right)$583.9993, found 583.9991. Anal. calcd. for $\mathrm{C}_{27} \mathrm{H}_{14} \mathrm{BrClN}_{6} \mathrm{O}_{3}$ (585.81): C, 55.36; H, 2.41; N, 14.35. Found C, 55.24; H, 2.49; N, 14.27.

[7-(4-Chlorophenyl)-6-(4-chloro-3-nitrophenylazo)-2-oxo-4-phenyl-1,2-dihydro[1,8]naphthyridine-3carbonitrile (24b). Recrystallized from EtOH/dioxane mixture (1:1) as pale brown crystals, yield: (2.21 g, 82\%), m.p.: above $300{ }^{\circ} \mathrm{C}$; IR (KBr): v/ $\mathrm{cm}^{-1} 3372(\mathrm{NH}), 2228(\mathrm{CN}), 1672(\mathrm{CO})$; ${ }^{1} \mathrm{H}-\mathrm{NMR}$ (DMSO- $\left.d_{6}\right): \delta=7.56-7.61(\mathrm{~m}, 4 \mathrm{H}, \mathrm{Ar}-\mathrm{H}), 7.65-7.68$ (m, 3H, Ar-H), 7.81-7.85 (m, 3H, Ar-H), 7.87-7.91 (m, 2H, 1 Ar-H and pyridine H4), $8.23(\mathrm{~d}, J=2.4 \mathrm{~Hz}, 1 \mathrm{H}, \mathrm{Ar}-\mathrm{H})$ and $13.45 \mathrm{ppm}(\mathrm{s}, 1 \mathrm{H}, \mathrm{NH})$; ${ }^{13} \mathrm{C}-\mathrm{NMR}\left(\mathrm{DMSO}-d_{6}\right): \delta=108.2,113.6,114.8,120.2,123.9,126.7,127.7,128.1,128.8,129.1,130.6$, 132.3, 132.9, 133.1, 134.7, 135.4, 140.6, 148.0, 150.3, 151.3, 159.3, 159.45 and 159.52 ppm (Ar-C, $\mathrm{CN}$ and CO); MS (EI): $m / z(\%) 541\left([\mathrm{M}+1]^{+}, 88.31\right), 540\left(\mathrm{M}^{+}, 100\right)$; HRMS (EI): $m / z$ calcd. for $\mathrm{C}_{27} \mathrm{H}_{14}{ }^{35} \mathrm{Cl}_{2} \mathrm{~N}_{6} \mathrm{O}_{3}\left(\mathrm{M}^{+}\right)$540.0498, found 540.0495.

\section{Conclusions}

The results of the study described above have led to the development of a simple approach for the synthesis of a novel class of pyridazin-3-one and 2-aminopyridine derivatives. Furthermore, the observations made during this work showed that these compounds are versatile precursors for the synthesis of some very important fused azines like azolo[1,5-a]pyrimidines and 1,8-naphthyridines, for which we expect a wide range of biological activity.

\section{Supplementary Materials}

Supplementary materials can be accessed at: http://www.mdpi.com/1420-3049/19/2/2637/s1.

\section{Acknowledgments}

Financial support for this study was provided by the University of Kuwait through a research grant (SC03/11). The facilities of Analab/SAF supported by research grants GS01/01, GS01/05, GS01/03 and GS03/08 are gratefully acknowledged.

\section{Conflicts of Interest}

The authors declare no conflict of interest.

\section{References and Notes}

1. Zhmurenko, L.A.; Molodavkin, G.M.; Voronina, T.A.; Lezina, V.P. Synthesis and antidepressant and anxiolytic activity of derivatives of pyrazolo[4,3-c]pyridine and 4-phenylhydrazinonicotinic acids. Pharm. Chem. J. 2012, 46, 15-19.

2. Smyth, L.A.; Matthews, T.P.; Horton, P.N.; Hursthouse, M.B.; Collins, I. Synthesis and reactivity of 3-amino-1H-pyrazolo[4,3-c]pyridin-4(5H)-ones: Development of a novel kinase-focussed library. Tetrahedron 2010, 66, 2843-2854. 
3. Suksrichavalit, T.; Prachayasittikul, S.; Nantasenamat, C.; Isarankura-Na-Ayudhya, C.; Prachayasittikul, V. Copper complexes of pyridine derivatives with superoxide scavenging and antimicrobial activities. Eur. J. Med. Chem. 2009, 44, 3259-3265.

4. Gaonkar, S.L.; Rai, K.M.L.; Prabhuswamy, B. Synthesis of novel 3-[5-ethyl-2-(2-phenoxy-ethyl)pyridin]-5-substituted isoxazoline libraries via 1,3-dipolar cycloaddition and evaluation of antimicrobial activities. Med. Chem. Res. 2007, 15, 407-417.

5. Butnariu, R.M.; Caprosu, M.D.; Bejan, V.; Mangalagiu, I.I.; Ungureanu, M.; Poiata, A.; Tuchilus, C.; Florescu, M. Pyridazine and phthalazine derivatives with potential antimicrobial activity. J. Heterocycl. Chem. 2007, 44, 1149-1152.

6. Kandile, N.G.; Mohamed, M.I.; Zaky, H.; Mohamed, H.M. Novel pyridazine derivatives: Synthesis and antimicrobial activity evaluation. Eur. J. Med. Chem. 2009, 44, 989-1996.

7. Hosni, H.M.; Abdulla, M.M. Anti-inflammatory and analgesic activities of some newly synthesized pyridinedicarbonitrile and benzopyranopyridine derivatives. Acta Pharm. 2008, 58, 175-186.

8. Dogruer, D.S.; Unlu, S.; Kupeli, E.; Banoglu, E.; Sahin, M.F. Synthesis of 2-[5,6-diphenyl-3(2H)pyridazinone-2-yl]acetamide and 3-[5,6-diphenyl-3(2H)-pyridazinone-2-yl]propanamide derivatives as analgesic and anti inflamematory agents. Turk. J. Pharm. Sci. 2007, 4, 57-70.

9. Gökçe, M.; Colak, M.S.; Küpeli, E.; Sahin, M.F. Synthesis and analgesic and anti-inflammatory activity of 6-phenyl/(4-methylphenyl)-3(2H)-pyridazinon-2-propionamide derivatives. Arzneim. Forsch. 2009, 59, 357-363.

10. Ali, M.A.; Yar, M.S.; Siddiqui, A.A.; Sriram, D.; Yogeeswari, P.; de Clercq, E. Synthesis and anti-HIV activity of $N^{\prime}$-nicotinoyl-3-(4'-hydroxy-3'-methylphenyl)-5-[substituted phenyl]-2pyrazo- lines. Acta Pol. Pharm. 2007, 64, 423-428.

11. Kumar, S.; Das, S.K.; Dey, S.; Maity, P.; Guha, M.; Choubey, V.; Panda, G.; Bandyopadhyay, U. Antiplasmodial activity of [(aryl)arylsulfanylmethyl]pyridine. Antimicrob. Agents Chemother. 2008, 52, 705-715.

12. Lourenço, M.C.S.; de Souza, M.V.N.; Pinheiro, A.C.; de Lima Ferreira, M.; Goncalves, R.S.B.; Nogueira, T.C.M.; Peralta, M.A. Evaluation of anti-tubercular activity of nicotinic and isoniazid analogues. ARKIVOC 2007, xv, 181-191.

13. Sharma, P.C.; Jain, S. Synthesis and in vitro antibacterial activity of some novel $\mathrm{N}$-nicotinoyl-1-ethyl-6-fluoro-1,4-dihydro-7-piperazin-1-yl-4-oxoquinoline-3-carboxylates. Acta Pol. Pharm. 2008, 65, 551-556.

14. Shafiee, A.; Rastkari, N.; Sharifzadeh, M. Anticonvulsant activities of new 1,4-dihydropyridine derivatives containing 4-nitroimidazolyl substituents. Daru 2004, 12, 81-86.

15. Rubat, C.; Coudert, P.; Refouvelet, B.; Tronche, P.; Bastide, P.; Bastide, J. Anticonvulsant activity of 3-oxo-5-substituted benzylidene-6-methyl-(4H)-2-pyridazinylacetamides and 2-pyridazinylacetyl-hydrazides. Chem. Pharm. Bull. 1990, 38, 3009-3013.

16. Chintakunta, V.K.; Akella, V.; Vedula, M.S.; Mamnoor, P.K.; Mishra, P.; Casturi, S.R.; Vangoori, A.; Rajagopalan, R. 3-O-Substituted benzyl pyridazinone derivatives as COX inhibitors. Eur. J. Med. Chem. 2002, 37, 339-347. 
17. Rathish, I.G.; Javed, K.; Bano, S.; Ahmad, S.; Alam, M.S.; Pillai, K.K. Synthesis and blood glucose lowering effect of novel pyridazinone substituted benzenesulfonylurea derivatives. Eur. J. Med. Chem. 2009, 44, 2673-2678.

18. Barbaro, R.; Betti, L.; Botta, M.; Corelli, F.; Giannaccini, G.; Maccari, L.; Manetti, F.; Strappaghetti, G.; Corsano, S. Synthesis, biological evaluation, and pharmacophore generation of new pyridazinone derivatives with affinity toward $\alpha_{1}$ - and $\alpha_{2}$-adrenoceptors. J. Med. Chem. 2001, 44, 2118-2132.

19. Joule, J.A.; Mills, K. Heterocyclic Chemistry, 5th ed.; Wiley Blackwell: Oxford, UK, 2010; pp. 645-664.

20. Atanasova. M.; Ilieva, S.; Galabov, B. QSAR analysis of 1,4-dihydro-4-oxo-1-(2-thiazolyl)-1,8naphthyridines with anticancer activity. Eur. J. Med. Chem. 2007, 42, 1184-1192.

21. Miguel, F.B.; Monica, C.M.L.G; Elena, P.M.; Berta, L.; de Beatriz, P.T.; Ana, R.; Nuria, A.; Francisco, L.; Dolores, M.M.; Olivier, L.; et al. Pyrazolo[3,4-c]pyridazines as novel and selective inhibitors of cyclin-dependent kinases. J. Med. Chem. 2005, 48, 6843-6854.

22. Malinka, W.; Redzicka, A.; Lozach, O. New derivatives of pyrrolo[3,4- $d]$ pyridazinone and their anticancer effects. Farmaco 2004, 59, 457-462.

23. Thapa, P.; Karki, R.; Thapa, U.; Jahng, Y.; Jung, M.-J.; Nam, J.M.; Na, Y.; Kwon, Y.; Lee, E.-S. 2-Thienyl-4-furyl-6-aryl pyridine derivatives: Synthesis, topoisomerase I and II inhibitory activity, cytotoxicity, and structure-activity relationship study. Bioorg. Med. Chem. 2010, 18, 377-386.

24. Thapa, P.; Karki, R.; Choi, H.; Choi, J.H.; Yun, M.; Jeong, B.-S.; Jung, M.-J.; Nam, J.M.; Na, Y.; Cho, W.-J.; et al. Synthesis of 2-(thienyl-2-yl or -3-yl)-4-furyl-6-aryl pyridine derivatives and evaluation of their topoisomerase I and II inhibitory activity, cytotoxicity, and structure activity relationship. Bioorg. Med. Chem. 2010, 18, 2245-2254.

25. Sotelo, E.; Fraiz, N.; Yanez, M.; Terrades, V.; Laguna, R.; Cano, E.; Ravina, E. Pyridazines. Part XXIX: Synthesis and platelet aggregation inhibition activity of 5-substituted-6-phenyl-3(2H)pyridazinones novel aspects of their biological action. Bioorg. Med. Chem. 2002, 10, 2873-2882.

26. Griebel, G.; Perrault, G.; Sanger, D.J. Differences in anxiolytic-like profile of two novel nonbenzodiazepine BZ (omega) receptor agonists on defensive behaviors of mice. Pharmacol. Biochem. Behav. 1999, 62, 689-694.

27. Wermuth, C.G.; Schlewer, G.; Bourguignon, J.J.; Maghioros, G.; Bouchet, M.J.; Moire, C.; Kan, J.P.; Worms, P.; Biziere, K. 3-Aminopyridazine derivatives with atypical antidepressant, serotonergic, and dopaminergic activities. J. Med. Chem. 1989, 32, 528-537.

28. Caliskan, E.B.; Sukuroglu, M.; Coban, T.; Banoglu, E.; Suzen, S. Screening and evaluation of antioxidant activity of some pyridazine derivatives. J. Enz. Inhib. Med. Chem. 2008, 23, 225-229.

29. Chen, K.; Kuo, S.-C.; Hsieh, M.-C.; Mauger, A.; Lin, C.M.; Hamel, E.; Lee, K.-H. Antitumor agents. 178. Synthesis and biological evaluation of substituted 2-aryl-1,8-naphthyridin-4(1H)ones as antitumor agents that inhibit tubulin polymerization. J. Med. Chem. 1997, 40, 3049-3056.

30. Zhang, S.-X.; Bastow, K.F.; Tachibana, Y.; Kuo, S.-C.; Hamel, E.; Mauger, A.; Narayanan, V.L.; Lee, K.-H. Antitumor agents. 196. Substituted 2-thienyl-1,8-naphthyridin-4-ones: Their synthesis, cytotoxicity, and inhibition of tubulin polymerization. J. Med. Chem. 1999, 42, 4081-4087. 
31. Wu, J.; Kang, S.; Luo, L.; Shi, Q.; Ma, J.; Yin, J.; Song, B.; Hu, D.; Yang, S. Synthesis and antifungal activities of novel nicotinamide derivatives containing 1,3,4-oxadiazole. Chem. Cent. J. 2013, 7, 64.

32. Behbehani, H.; Ibrahim, H.M.; Elnagdi, M.H. Non-concerted nucleophilic [4+1] cycloaddition of (dimethylamino)methoxycarbene to arylazonicotinates in the synthesis of pyrazolo[3,4c]pyridines and pyrazolo[4',3':4,5]pyrido[2,3-d]pyrimidines. Tetrahedron 2013, 69, 6176-6184.

33. Behbehani, H.; Ibrahim, H.M.; Makhseed, S.; Mahmoud, H. Applications of 2-arylhydrazononitriles in synthesis: Preparation of new indole containing 1,2,3-triazole, pyrazole and pyrazolo[1,5a]pyrimidine derivatives and evaluation of their antimicrobial activities. Eur. J. Med. Chem. 2011, 46, 1813-1820.

34. Behbehani, H.; Ibrahim, H.M.; Makhseed, S.; Elnagdi, M.H.; Mahmoud, H. 2-Aminothiophenes as building blocks in heterocyclic synthesis: Synthesis and antimicrobial evaluation of a new class of pyrido[1,2-a]thieno[3,2-e]pyrimidine, quinoline and pyridin-2-one derivatives. Eur. J. Med. Chem. 2012, 52, 51-65.

35. Behbehani, H.; Ibrahim, H.M. 4-Thiazolidinones in heterocyclic synthesis: Synthesis of novel enaminones, azolopyrimidines and 2-arylimino-5-arylidene-4-thiazolidinones. Molecules 2012, 17, 6362-6385.

36. Behbehani, H.; Ibrahim, H.M. Organocatalysis in heterocyclic synthesis: DABCO as a mild and efficient catalytic system for the synthesis of a novel class of quinazoline, thiazolo[3,2- $a$ ]quinazoline and thiazolo[2,3-b]quinazoline derivatives. Chem. Cent. J. 2013, 7, 82.

37. Ibrahim, H.M.; Behbehani, H.; Elnagdi, M.H. Approaches towards the synthesis of a novel class of 2-amino-5-arylazonicotinate, pyridazinone and pyrido[2,3-d]pyrimidine derivatives as potent antimicrobial agents. Chem. Cent. J. 2013, 7, 123.

38. Crystallographic data for 3d (ref. CCDC 982246) can be obtained on request from the director, Cambridge Crystallographic Data Center, 12 Union Road, Cambridge CB2 1EW, UK.

39. Crystallographic data for 31 (ref. CCDC 982247) can be obtained on request from the director, Cambridge Crystallographic Data Center, 12 Union Road, Cambridge CB2 1EW, UK.

40. Behbehani, H.; Ibrahim, H.M. A strategy for the synthesis of 2-aryl-3-dimethylaminopyrazolo[3,4-c]pyridines that utilizes [4+1] cycloaddition reactions of 5-arylazo-2,3,6-trisubstituted pyridines. Tetrahedron. 2013, 69, 10535-10543.

41. Al-Mousawi, S.M.; Moustafa, M.S.; Abdelshafy, I.A.; Elnagdi, M.H. Reassignment of the structures of condensation products of $\alpha$-keto $\alpha$-formylarylhydrazones with ethyl cyanoacetate: A novel route to ethyl 5-arylazo-2-hydroxynicotinates. Tetrahedron Lett. 2011, 52, 202-204.

42. Crystallographic data for $\mathbf{1 8}$ (ref. CCDC 943164) can be obtained on request from the director, Cambridge Crystallographic Data Center, 12 Union Road, Cambridge CB2 1EW, UK.

43. Sheldrick, G.M. A short history of SHELX. Acta Cryst. 2008, A64, 112-122.

Sample Availability: Samples of all compounds are available from the authors.

(C) 2014 by the authors; licensee MDPI, Basel, Switzerland. This article is an open access article distributed under the terms and conditions of the Creative Commons Attribution license (http://creativecommons.org/licenses/by/3.0/). 